\title{
Gradhiva
}

GRADHI

Revue d'anthropologie et d'histoire des arts

28 | 2018

Capsules temporelles

\section{Des pièces d'époque aux capsules temporelles. Temps historique et temps vécu dans l'expérience esthétique}

From period rooms to time capsules. Historical time and lived time in aesthetic

experience

\section{Rémi Labrusse}

\section{OpenEdition}

Journals

Édition électronique

URL : http://journals.openedition.org/gradhiva/3721

DOI : 10.4000/gradhiva.3721

ISSN : 1760-849X

\section{Éditeur}

Musée du quai Branly Jacques Chirac

\section{Édition imprimée}

Date de publication : 28 novembre 2018

Pagination : 76-111

ISBN : $978-2-35744-111-8$

ISSN : 0764-8928

\section{Référence électronique}

Rémi Labrusse, « Des pièces d'époque aux capsules temporelles. Temps historique et temps vécu dans l'expérience esthétique », Gradhiva [En ligne], 28 | 2018, mis en ligne le 28 novembre 2020, consulté le 07 janvier 2021. URL : http://journals.openedition.org/gradhiva/3721 ; DOI : https:// doi.org/10.4000/gradhiva.3721 


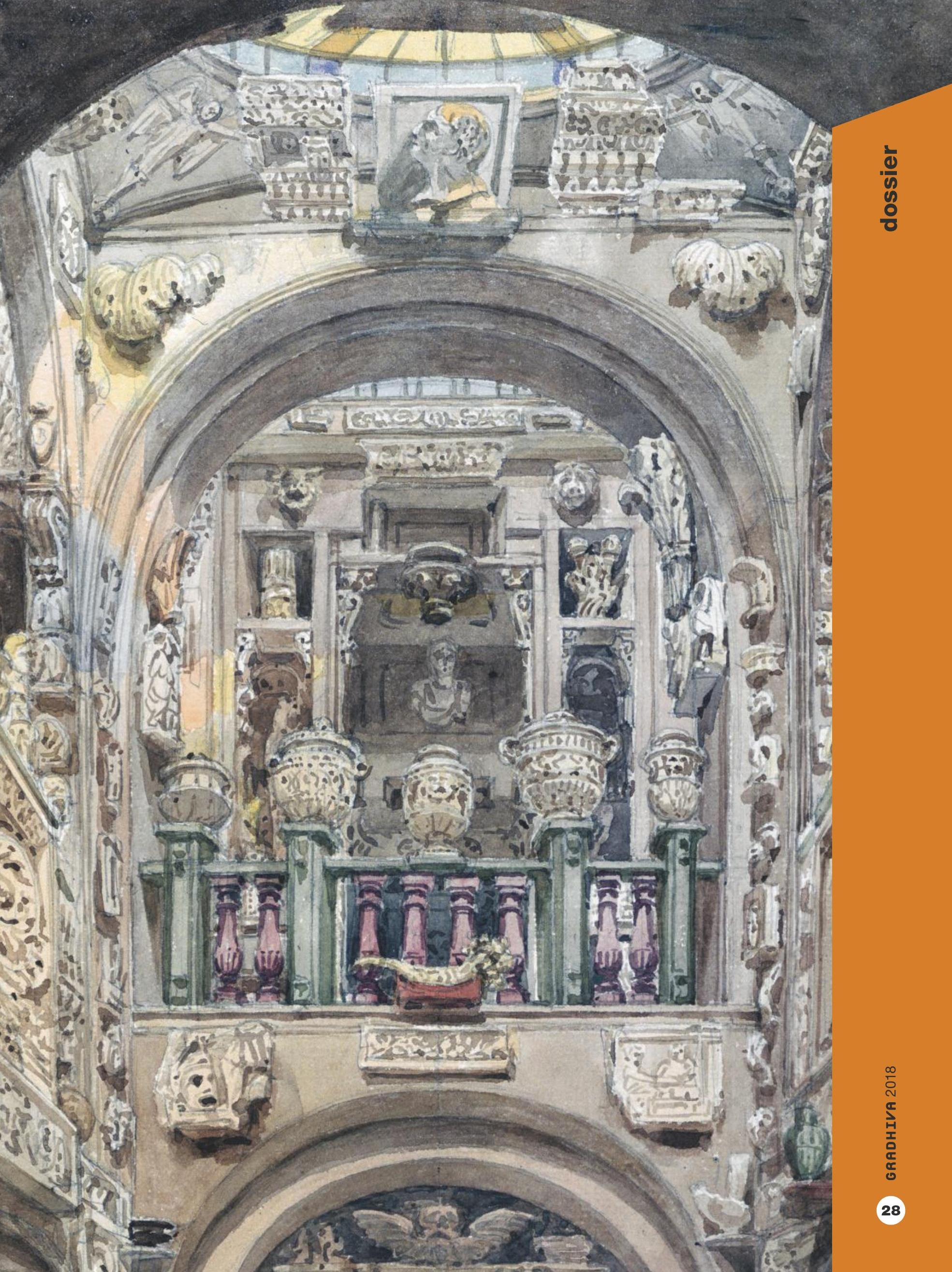


Des pièces d'époque aux capsules temporelles

Temps historique et temps vécu dans l'expérience esthétique

par Rémi Labrusse

Les pièces d'époque, ou period rooms, se définissent comme des transplantations ou des réinventions d'intérieurs historiques dans un cadre muséal. Capsules dans la capsule du musée, elles ont pour mission d'y sauvegarder le sentiment d'un temps vécu, celui de l'habitation et de ses usages, et de réparer ainsi l'impression macabre qui a plané dès l'origine sur l'idée de musée. Concrètement, cependant, l'expérience de la pièce d'époque opère un renversement de cet horizon utopique: les dimensions du temps, en s'emmêlant, s'y donnent sur le mode de la perte, suivant un processus qui, selon le psychanalyste et phénoménologue Ludwig Binswanger, est le propre de la mélancolie. Troisième temps, enfin: l'encapsulage muséal donne une profondeur réflexive à cet affect mélancolique. Par là, la pièce d'époque devient aussi l'espace d'accomplissement d'un travail de deuil à l'égard des ambitions propres à toute fabrication de capsule temporelle.

mots clés

period room, musée, mélancolie,

temporalité, Binswanger 
1. Michel Leiris,

"Glossaire: j'y serre mes gloses", La Révolution surréaliste $6,1^{\text {er }}$ mars 1926: 20-21, ici 21.

2. Raymond Montpetit, pour sa part, a proposé l'expression «intérieur d'époque". Voir Montpetit 1996: 55-103, ici 70.
«VIE - un dé la sépare du vide ${ }^{1}$ »

\section{Une histoire de musée?}

En son sens le plus étroit, la pièce d'époque ${ }^{2}$ - traduction naturelle du syntagme anglais period room - désigne un intérieur arraché à un lieu d'habitation, revêtements muraux et meubles compris, et réinstallé dans un musée pour y représenter un pan de l'histoire des formes, des usages et des mœurs. Historique et muséale, la pièce d'époque stricto sensu est une pièce détachée, passée de l'usage, dans un environnement de vie quotidienne, à la contemplation, dans un environnement muséal. L'opération de transposition dont elle est le résultat implique toujours une part d'invention, afin d'adapter au mieux l'installation d'ensemble à son nouveau but, lequel consiste à rendre immédiatement sensible le temps passé. Même lorsque, suivant la distinction opérée par Edward Porter Alexander, la pièce d'époque est plutôt artistique qu'historique, l'espace s'y offre en effet à la vision, primordialement, comme une figure du temps, qu'il matérialise et donne à éprouver physiquement (Alexander 1964). Comme on parle en français d'objet d'époque, sans plus de précision, on pourra dire de la pièce d'époque qu'elle s'individualise matériellement pour donner à celui qui la rencontre un sentiment de la temporalité en tant que telle.

Cette définition stricte présente des possibilités d'extension, en amont, avec les environnements historicistes dans des espaces habités (fumoirs orientalistes, chambres à coucher néogothiques, salons pseudo-Art déco, etc.) et, en aval, avec les "period settings", suivant l'expression de Dianne Pilgrim, ces installations muséales qui évoquent un moment historique (fig. 1) et prennent la forme d'une pièce ou d'un coin de pièce sans pour autant avoir constitué explicitement, auparavant, un lieu d'habitation réel (Pilgrim 1978). Mais dans tous les cas, à des degrés divers d'intensité, l'horizon de sens demeure toujours une matérialisation spatiale du temps, dont l'effet est conditionné par son intégrité, qu'il s'agit de préserver autant que faire se peut. Sous cette lumière, la pièce d'époque relève intrinsèquement de l'esprit muséal, qu'elle appartienne ou non effectivement à un musée. Elle incarne et même elle semble magnifier le désir de scellement qui, dès son origine au $\mathrm{XVIII}^{\mathrm{e}}$ siècle, hante l'idée de musée. Concurrençant l'idéologie de l'accès public et de la libre fréquentation, le musée chérit en effet sa propre clôture, veut l'encapsulement des choses qu'il recèle une fois pour toutes comme le ferait un mausolée. Le personnage du conservateur, dont Benjamin Zix a dessiné le portrait allégorique en imaginant Dominique Vivant-Denon, premier directeur du musée Napoléon, au fond des caves de son nouveau sanctuaire (fig. 2.), y est un encapsuleur, gardien toujours un peu jaloux d'une pyramide à l'intérieur de laquelle il orchestre la mise en ordre des témoins matériels de l'histoire, pour un semblant d'éternité. Et que nul n'y pénètre donc, de peur de déranger cet ordre des choses.

Ainsi en irait-il, superlativement, de la pièce d'époque, capsule dans la capsule, où le temps historique s'enrichirait d'une dimension subjective miraculeusement quintessenciée. Au cœur de ce dispositif allégorique du triomphe de l'Occident capitaliste qu'est le musée, la pièce d'époque permettrait à ce dernier de se parfaire en se dégageant des accusations répétées qui, dès sa naissance, l'ont assimilé à un lieu macabre, privilégiant la matière morte sur les usages de la vie. La capsule, quoique toujours marquée par d'infranchissables limites, n'y serait pas conçue sur le mode du sarcophage mais 
Des pièces d'époque aux capsules temporelles. Temps historique et temps vécu dans l'expérience esthétique. Par Rémi Labrusse

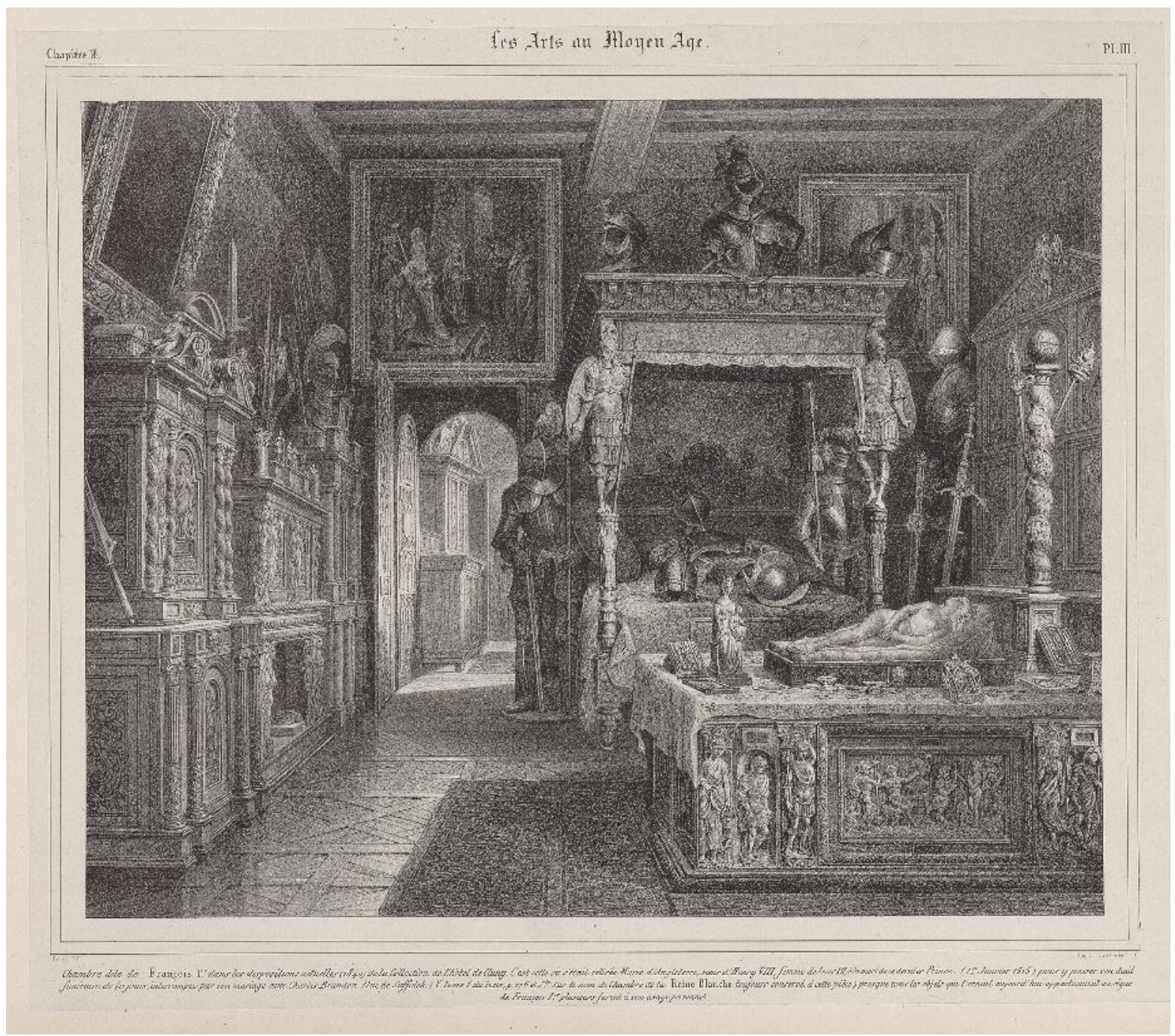

fig. 1

"Chambre dite de François

|er dans les dispositions

actuelles (1840) de la

Collection de l'hôtel de

Cluny », pl. II du chapitre ॥

de l'ouvrage d'Alexandre

du Sommerard, Les Arts

au Moyen Âge, Paris.

Hôtel de Cluny et Techener,

1838-1846

(C) RMN-Grand Palais

(musée de Cluny - musée

national du Moyen Âge)

/ image musée Cluny. 


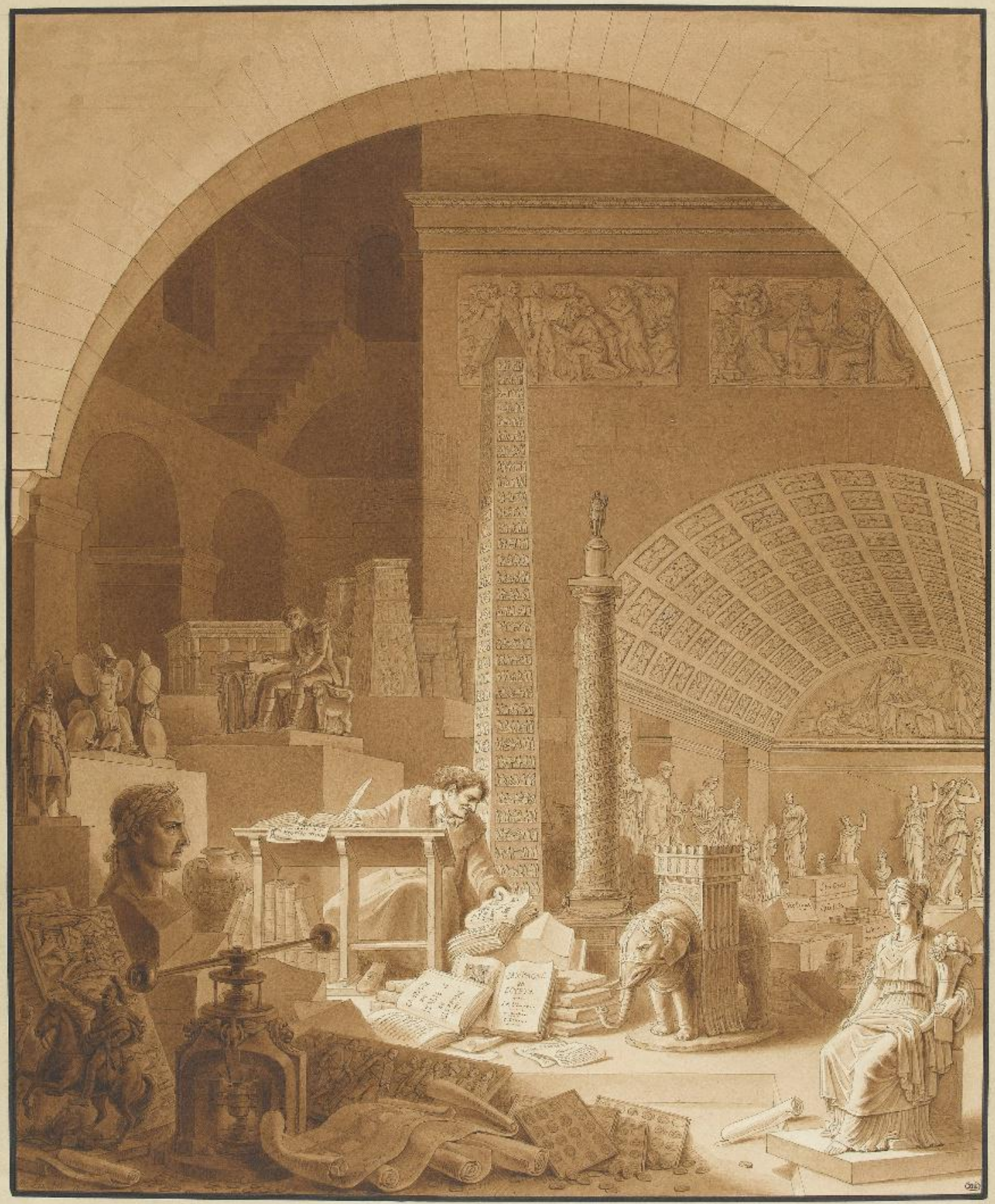


plutôt sur celui du berceau protecteur d'une vie mystérieusement perpétuée. C'est d'ailleurs pourquoi, au sens large, les pièces d'époque naissent à peu près exactement au même moment que les musées modernes: dès le musée des Monuments français d'Alexandre Lenoir, à Paris dans les années 1800, on en voit s'esquisser les effets de sens, même si, au sens strict, les pièces détachées et déplacées, ces «moving rooms » auxquelles John Harris a consacré un livre, sont un phénomène plus tardif, dont les États-Unis de la fin du $X I X{ }^{e}$ et de la première moitié du $X X^{e}$ siècle se sont fait une spécialité (Harris 2007).

Pour mieux comprendre cette relation originelle, quoique dialectique, entre musée et pièce d'époque, il faut d'abord préciser le régime temporel si particulier auquel l'un et l'autre se rattachent initialement. De quel temps parlet-on lorsqu'on décide d'identifier l'idée de «période» ou d'“époque » à celle d'un espace clos, exalté sous les espèces de la «pièce» ou de la «chambre»? La notion de période - qui trône au fondement de la period room - arrime le décompte de la durée au cheminement des astres, à leur « route», pour suivre l'étymologie grecque du mot (Labrusse 2015): elle désigne un temps cosmologique, désubjectivisé, soumis à la loi des nombres, qu'une vision scientifique du monde a voulu, à partir de la Renaissance, ériger en principe ordonnateur de la temporalité. Époque, d'ailleurs, dit aussi cela, en insistant sur la stabilité plutôt que sur le mouvement réglé: l'épochè grecque, encore une fois, c'est le suspens, la parenthèse, l'arrêt, étendue nettement délinéée d'espacetemps, «point fixe dans l'histoire dont on se sert dans la chronologie», comme l'écrit le Dictionnaire de l'Académie française dans son édition de $1740^{3}$ - l'envers, en somme, de l'insaisissable durée. Les trois articles de l'Encyclopédie consacrés à la notion, qui était encore en cours de formation au $\mathrm{XVIII}$ e siècle, sont tout aussi explicites; sous la plume de l'abbé Edme-François Mallet, au début des années 1750, l'approche historique, directement plagiée de Bossuet, arrive en dernier et elle procède des deux premières approches, en logique et en astronomie: «Ce mot vient d'un mot grec qui signifie s'arrêter, parce que les époques dans l'histoire sont comme des lieux de repos, et pour ainsi dire, des stations où l'on s'arrête pour considérer de-là plus à son aise ce qui suit et ce qui précède, et pour lier entre eux les événements. » (Mallet 1755: 834-836) Enfin, à peu près au même moment, la signification du siècle achève de se dépouiller de ses connotations métaphysiques de très longue durée (le «saecula saeculorum » de la Vulgate) pour désigner une unité temporelle fixe de cent années, scandant mathématiquement le cours de l'histoire et exigeant de la vie foisonnante des sociétés qu'elle y obéisse ${ }^{4}$.

Mais ce double mouvement de mathématisation et de spatialisation du temps des sociétés humaines - c'est-à-dire, aussi bien, cette articulation de l'histoire et de la cosmologie - ne s'est pas fait aussi harmonieusement qu'on aurait pu le rêver. On sent bien que la rationalité qui se déploie dans les rouages de la grande horloge de l'univers devient incontrôlablement «arbitraire » sous le ciel des hommes: «Comme il n'y a point de raisons tirées de l'Astronomie qui rendent l'une préférable à l'autre, la fixation des époques est purement arbitraire. [...] II n'y a aucune de ces époques qui ne soit le sujet de quelque dispute, tant il y a d'incertitude dans la doctrine des temps. " (Ibid. : 834-835) Autant dire qu'au moment même où il s'imposait dans la conscience collective et dans le langage commun, le dur dénombrement du temps en périodes, en époques ou en siècles a été souterrainement
3. À partir de l'édition de 1832-1835 apparaît un second sens : "Il se dit aussi de toute partie du temps considérée par rapport à ce qui s'y passe, à ce qu'on y fait. " Dès 1861, Bossuet, au début de son Discours sur l'histoire universelle (repris par Littré dans son Dictionnaire en 1874) justifiait ainsi l'étymologie du mot "époque»:

"d'un mot grec qui signifie s'arrêter, parce qu'on s'arrête là, pour considérer comme d'un lieu de repos tout ce qui est arrivé devant ou après, et éviter par ce moyen les anachronismes, c'est-à-dire cette sorte d'erreur qui fait confondre les temps.»

4. Le milieu du XVIII ${ }^{e}$ siècle constitue une charnière. En 1751, par exemple, dans Le Siècle de Louis XIV, Voltaire n'associe pas le "siècle " à un décompte de cent années mais propose de distinguer "quatre siècles dans l'histoire du monde »: celui de "Philippe et d'Alexandre", celui de "César et d'Auguste", celui de «la gloire de l'Italie " et enfin celui de Louis XIV, scandé par le couronnement en 1643, la prise du pouvoir en 1661 et la mort du roi en 1715.

\section{ci-contre}

fig. 2

Benjamin Zix,

Portrait allégorique de Dominique

Vivant-Denon, 1811.

Plume et lavis sur papier, $42,2 \times 34,4 \mathrm{~cm}$,

Paris, musée du Louvre (c) RMN-Grand Palais

(musée du Louvre) / Thierry Le Mage. 
perçu sur le mode de la perte: on y éprouvait la vaine tentative d'imprimer à la chronologie ainsi produite une légitimité ontologique dont on reconnaissait par ailleurs qu'elle lui ferait toujours irrémédiablement défaut. Du coup, l'histoire qui en découle, avec ses divisions mathématiques en base décimale, est désignée comme une histoire non seulement «arbitraire» mais abstraite, où s'effondre l'expérience intime "des profondeurs temporelles", selon l'expression de Reinhart Koselleck (1990 [1979] : 266).

L'ère industrielle se fit, par excellence, la caisse de résonance de ce sentiment de perte, l'âge où «[l]a temporalisation qui, au départ, ne s'inscrivait qu'au sein de la théorie de l'histoire, pénétrait en profondeur la vie quotidienne " (ibid. : 294) et y semait l'angoisse. Enserrés dans les cadres de la périodisation, ce temps figé dans le cristal des nombres, les simples actes de l'existence - et non plus seulement les grands événements dynastiques et militaires - se vivaient désormais, plus ou moins dramatiquement, comme les jouets d'un temps historique sans épaisseur, voué à l'inauthenticité, au déracinement. En retour se sont manifestées avec toujours plus d'insistance les revendications du moi en faveur d'un temps subjectif dont l'informulable vérité originaire rôde désormais comme un remords dans les consciences: soit sur un mode mélancolique, par le déploiement d'un imaginaire du temps passé où récits rêvés et factuels se mêlent - I'historicisme -, soit sur un mode vitaliste, par l'exploration d'une temporalité intime, indénombrable et inspatialisable, jaillissant de l'obscurité des affects. Les approches historiques se modifient, on commence à dénoncer l'histoire des dynasties et des batailles au profit de l'histoire des pratiques quotidiennes et des relations inter-personnelles; la psychologie - bientôt relayée par la psychanalyse, le vitalisme bergsonien et surtout la phénoménologie - s'intéresse à la structuration originaire, dans chaque conscience individuelle, de la temporalité.

Plus qu'aucun autre champ de la pensée, l'histoire de l'art s'est trouvée - et continue d'être - déchirée par ce conflit fondamental entre temps périodisé et temps vécu. D'un côté, la spatialité mathématique des périodes promettait d'assurer à la jeune discipline une légitimité scientifique appuyée sur l'exemple prestigieux de l'histoire générale; elle conférait un cadre stable à un grand récit qui s'était formulé d'emblée sur le mode de la rupture entre des blocs de temps - Antiquité et modernité, âges sombres et Renaissance, classicisme et baroque, etc.; enfin, elle semblait convenir admirablement à un savoir qui s'attachait essentiellement à des objets et se donnait pour tâche de les classer, conduisant donc naturellement à exiger de ses praticiens l'invention d'une taxinomie. D'un autre côté, cependant, l'œuvre d'art, plus qu'aucun autre fait de la culture, manifestait sa propension à déborder les cadres de l'histoire qui l'accueillait sans pouvoir la circonscrire: elle incarnait la capacité de l'intelligence créatrice à trouer l'histoire, son refus d'être cantonnée à l'expression d'une époque. Ou du moins, à tort ou à raison, allait-on chercher cela dans l'œuvre d'art, cette évidence d'une respiration outre-temps. D'où, au total, un déchirement qui a fait de l'histoire de l'art le lieu mental où, par excellence, se démultipliaient à l'envi les périodes, souspériodes, transitions, etc., et celui où l'approche historique, ainsi rigidifiée, était sans cesse ébranlée par l'attrait de réflexions transversales - on dit aujourd'hui volontiers «trans-périodes" -, nourries par l'anthropologie ou la philosophie esthétique. 
Concrètement, le musée moderne, prenant son essor de concert avec la discipline académique de l'histoire de l'art au début du XIX $X^{\mathrm{e}}$ siècle, a paru devoir constituer la plus impeccable incarnation de ce temps spatialisé appliqué à l'histoire, par le moyen des œuvres d'art. Qu'il s'agisse de la Grande Galerie du Louvre, réorganisée par Dominique-Vivant Denon de manière à ce que l'avancée pas à pas du visiteur constitue en soi un cours d'histoire de l'art, ou du musée des Monuments français d'Alexandre Lenoir, dans lequel chaque salle devait être dédiée à un siècle, du XIII à nos jours, l'espace muséal matérialisait le plus exactement possible les divisions rationnelles du temps: les époques, les siècles.

Sauf qu'aussitôt, des voix se sont fait entendre pour dénoncer ce qu'on estimait mortifère dans cette surprenante entreprise, à savoir la mise en espace d'un grand récit prétendument rationnel dont les époques étaient les chapitres, les écoles les phrases et les œuvres les mots. Pour formuler ces critiques, pour signifier cette angoisse d'une désagrégation du temps vivant dans les salles de musées, pour stigmatiser la nature factice et morbide du temps muséal, c'est la métaphore du cimetière qui a le plus souvent été mobilisée: «véritable cimetière des arts», disait déjà Antoine Chrysostome Quatremère de Quincy en 1801, à propos du dépôt des Petits-Augustins, futur musée des Monuments français, où la « foule [des] objets » était « désormais sans rapport avec les idées qui leur donnaient la vie» (Quatremère de Quincy 1910: 182). II eut des suiveurs sans nombre, de Théophile Thoré en 1861 - «Les musées ne sont que les cimetières de l'art » (Thoré 1870: 84) - à Filippo Tommaso Marinetti en 1909 - «Musées, cimetières!... Identiques vraiment dans leur sinistre coudoiement de corps qui ne se connaissent pas ${ }^{5}$ » (Marinetti 1979: 153) -, en passant par Edmond de Goncourt qui refusait de voir les «choses d'art qui ont fait le bonheur de [sa] vie» finir dans «la froide tombe d'un musée " 6 . La mort paraissait là à l'œuvre, dans tous les sens du terme, non seulement parce que le passé y prévalait sur le présent, mais surtout parce que ce passé lui-même s'y présentait comme désincarné, conservé sous forme de dépouilles, traces offertes à l'autopsie sous le scalpel de la raison critique, mais définitivement muettes, estimait-on, pour le dialogue vivant avec le sentiment esthétique du spectateur. L'encapsulage extraordinairement méticuleux que manifestent tous les actes de la conservation préventive (avec, à son horizon idéal, l'annulation de toute agitation humaine), loin de promettre une survie, n'y aurait été rien d'autre qu'une mise au tombeau.

Au même moment, tout se passe comme si l'invention et le développement de la period room (de la pièce d'époque) avaient reflété ces tensions, s'affairant à réparer, dans le champ muséal, un sentiment désastreux de perte du temps subjectif et à faire droit aux revendications d'une autre temporalité, celle de la durée vécue. Déjà, à leur manière, les salles du musée des Monuments français cherchaient à conférer une résonance émotionnelle immédiate au didactisme de leur ordonnancement par siècles, par la constitution d'environnements immersifs. Au moment où «le particulier fait son entrée dans l'histoire », comme l'a perçu Walter Benjamin, commencent aussi les «fantasmagories de l'intérieur » (1991 [1939]: 298). Lorsqu'elles ont pénétré vraiment dans l'enceinte des musées - ce fut donc aux États-Unis, au début $\mathrm{du} x \mathrm{x}^{\mathrm{e}}$ siècle -, les period rooms ont formulé la proposition - le rêve - d'une miraculeuse présence du passé, réconciliant le temps périodisé et le temps vécu, le temps historique et le temps subjectif, l'époque mathématiquement définie et le flux intime de la durée: hyper-capsules, en quelque
5. Le Premier Manifeste du futurisme de Filippo Tommaso Marinetti a été publié pour la première fois à Paris dans Le Figaro du 20 février 1909.

6. Edmond de Goncourt, dédicace au catalogue de sa vente après décès: "Collection des Goncourt - Gravures du XVIII siècle" vente à Paris, hôtel Drouot, 26-28 avril 1897, Paris, A. Danlos, 1897, s. p. 


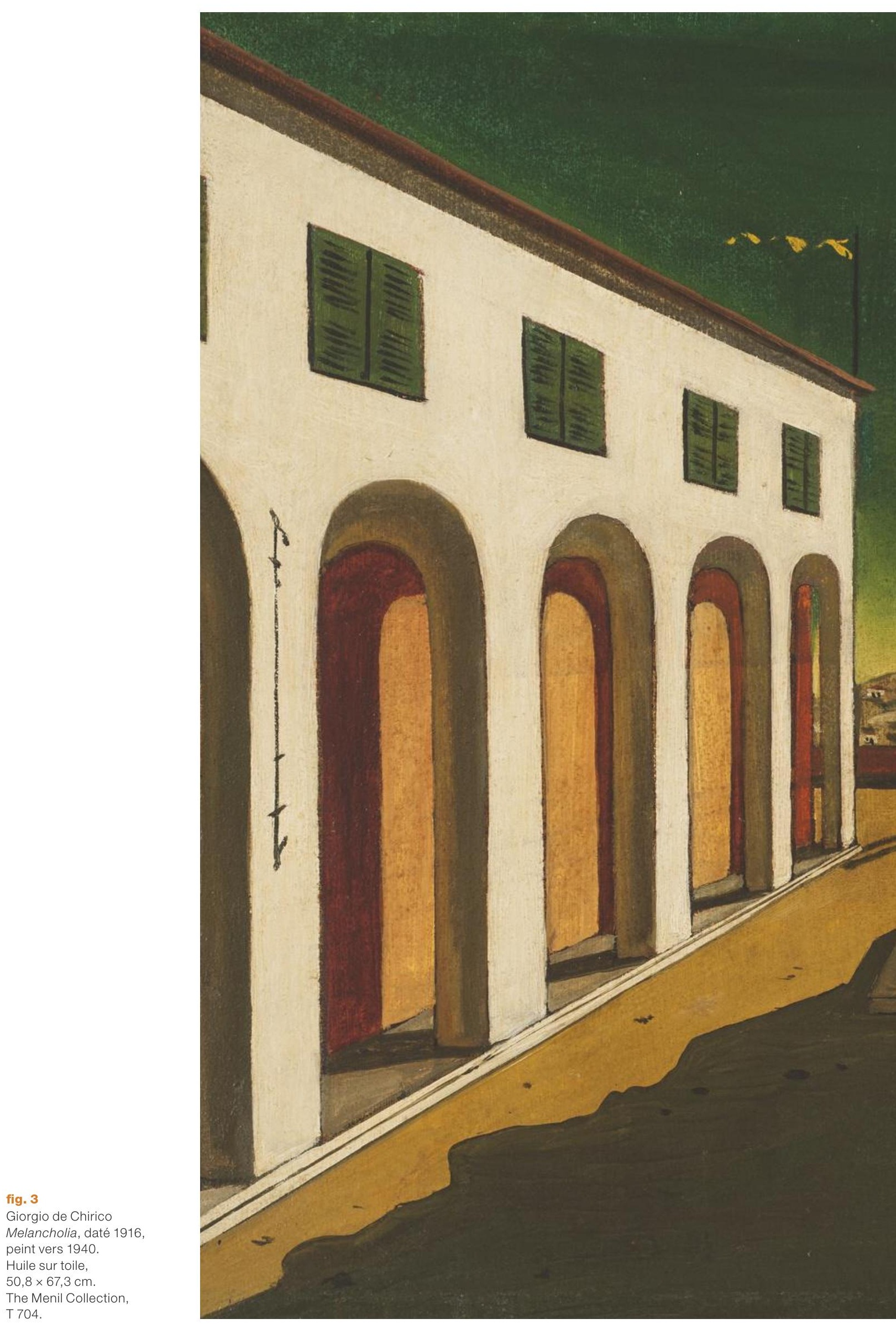

fig. 3

Giorgio de Chirico

T 704 . 


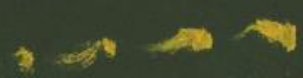

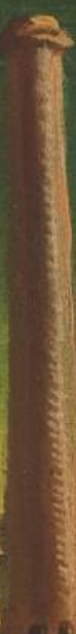

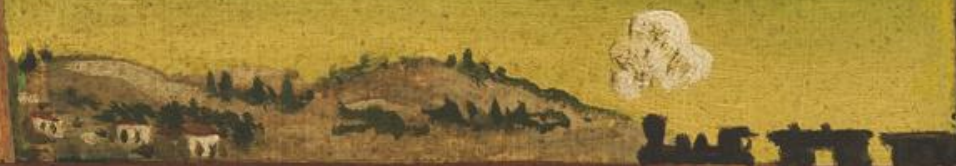
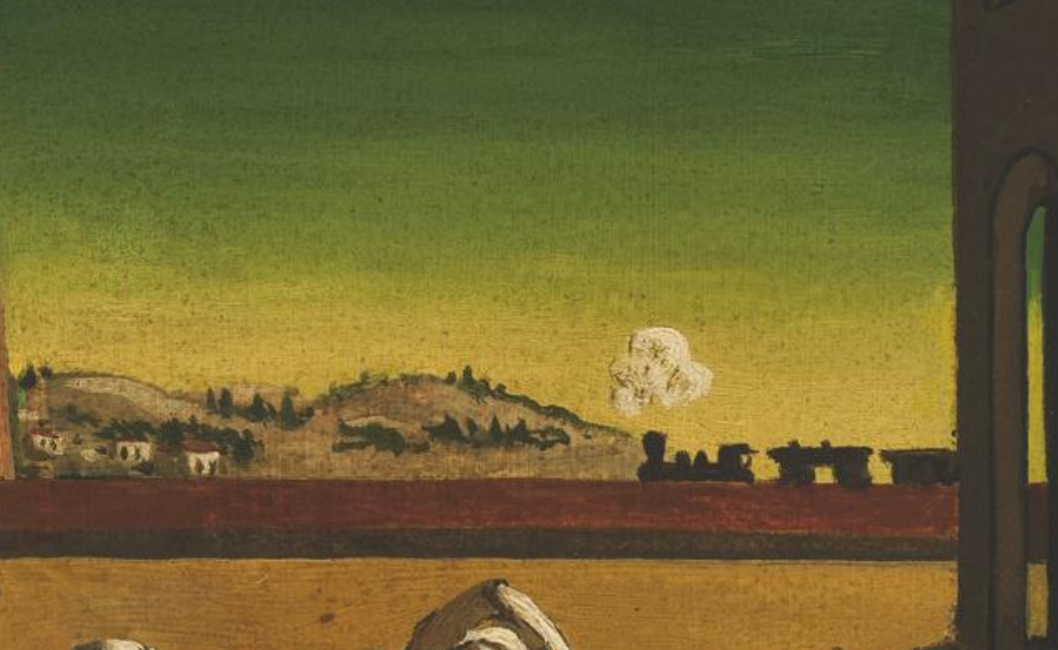

I col $1 x^{2}$.

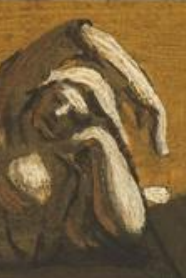


7. Dow 1922: 18, je traduis. George F. Dow est également cité dans Pilgrim 1978, p. 8 : "An effort was made to heighten the illusion of actual human occupancy by casually placing on the table before the fireplace in the parlor, a Salem newspaper printed in the year 1800 and on it a pair of silver-bowed spectacles, as though just removed by the reader. Elsewhere was placed a work basket with a half-knitted stocking on the top of other work, the knitting needles in place; and in other ways the illusion of daily occupancy was created.

8. Royal Cortissoz est également cité dans Kourelis 2007: 391-442, et plus particulièrement 406.

9. Réclamée à l'époque par le critique du New York Times, cette galerie n'a jamais été réalisée.

10. Compte rendu de la réouverture des salles par Kaufman 2007 : 24. sorte, aspirant à triompher des faiblesses originelles de leurs propres racines muséales. Y sont-elles parvenues? Leur destin ambigu, entre rejet et fascination, ainsi que la mélancolie qu'elles dispensent, lieux trop parfaits prêts pour une vie qui n'y aura plus jamais lieu, inclinent plutôt à penser qu'elles ont aiguisé la conscience critique d'une inadéquation entre période et usages de la vie, tels que les choses en portent la marque - écart, abîme dont l'histoire de l'art a pour vraie fonction de creuser l'énigme.

\section{Capturer la vie}

Qu'est-ce qui vit, qu'est-ce qui se produit, qu'est-ce qui a lieu dans ces petits mondes trop bien dépoussiérés et généralement baignés, derrière leurs fenêtres de papier, d'une lumière glauque qui ne donne du jour naturel que l'évidence de son absence? L'objectif a été défini avec une précision particulière dans ce contexte américain du début du $x x^{e}$ siècle, lorsque se sont multipliés les procédés de suggestion de ce que Charles B. Hosmer a nommé une impression de «vivabilité» («liveableness») (1965: 215-216). À Salem, par exemple - l'un des tout premiers musées à installer des pièces d'époque américaines, dès 1907 -, George Francis Dow, leur conservateur, raconte comment:

On avait fait un effort pour intensifier l'illusion d'une présence humaine effective, en plaçant négligemment sur la table devant la cheminée de l'entrée un journal de Salem imprimé en 1800, avec par-dessus une paire de lunettes à monture d'argent, comme si le lecteur venait de les laisser là; ailleurs, on avait mis une corbeille à ouvrage, avec dedans un bas à moitié tricoté au-dessus d'autres ouvrages, et les aiguilles à tricoter à leur place; et par d'autres moyens encore on créait l'illusion d'une présence quotidienne ${ }^{7}$.

À Brooklyn en 1929, le critique de l'Albany Times Union parle d'un «air chaleureusement humain» («warmly human air») qui flotte dans les «intérieurs " d'époque récemment inaugurés du musée (Murtha 2005: 211). Quant à Royal Cortissoz, l'influent critique du New York Herald Tribune et du Scribner's Magazine, il se félicite en 1924 que l' «American Wing» du Metropolitan Museum of Art de New York, bien que fondée sur «la recherche archéologique », donne avant tout à voir "de chaleureuses choses humaines» («warm human things») (Cortissoz 1925: 105) ${ }^{8}$. Le thème de la chaleur vitale est métaphorisé, dans un grand nombre de pièces d'époque, par la présence d'une cheminée (fig. 4), où l'on dispose volontiers des bûches encore intactes, comme pour conjurer sa toujours menaçante transformation en bouche d'ombre propice aux fantômes. Le même désir de suggestion de la vie se marque aussi dans l'association de sons aux objets: appels à créer une «galerie sonore» ("sound gallery») au Museum of the City of New York en $1930^{9}$ (Page 1999: 58); chants médiévaux diffusés dans les Barnard Cloisters à New York, à partir de 1914 (Kourelis 2007: 406; Harris 2007: 165); ou, tout récemment encore, bruits de conversation, de cheminée, de jeux de société et de chevaux passant sur la chaussée, dans les salles du XVIII ${ }^{\mathrm{e}}$ siècle français au Detroit Institute of Art ${ }^{10}$.

Par tous ces procédés, c'est la stricte objectalité, considérée comme mortifère, des choses détachées de leurs usages, qu'on s'efforce de contrebalancer par une impression de vie fluctuante, comme si la pièce d'époque 
Des pièces d'époque aux capsules temporelles. Temps historique et temps vécu dans l'expérience esthétique.

Par Rémi Labrusse

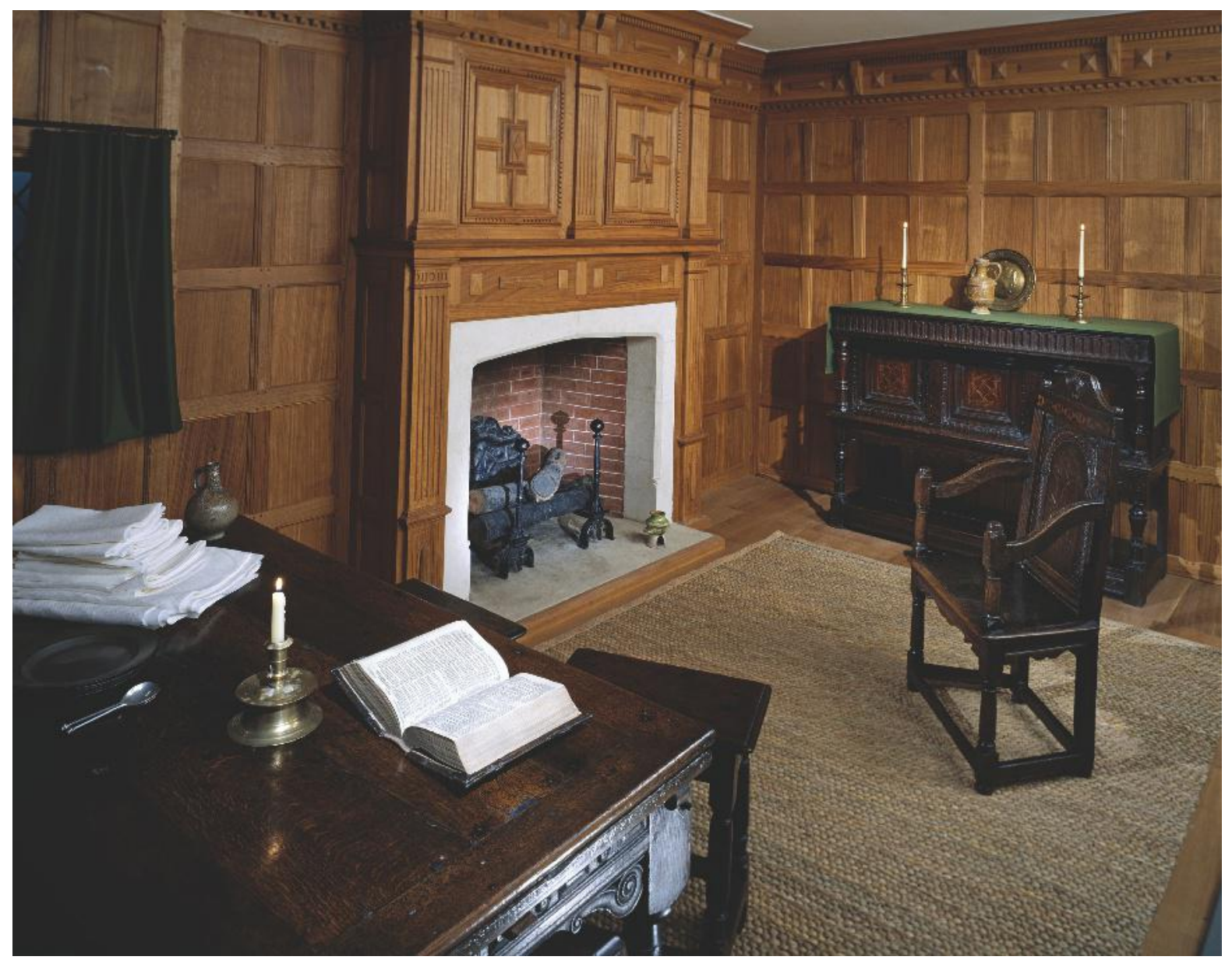

fig. 4

Le " 1630 Hall» au Geffrye

Museum, Londres.

(c) Geffrye Museum,

Londres. Photo

Christopher Ridley. 
11. Voir également Poe $1840: 243-245$ avait pour mission d'effacer les effets pervers du passage de la valeur d'usage à la valeur d'exposition. Loin de remettre en cause l'ambition intrinsèquement muséale d'encapsulage - comme aurait dit Andy Warhol (Görner [dir.] 2003) - du temps entre des murs, elle prétend parfaire l'opération en enrichissant la chronologie objective d'une dimension subjective. Pour répondre à l'angoisse récurrente d'un grand macabre muséal, elle se fixe d'orchestrer la fusion entre deux régimes de temporalité hétérogènes.

Ce faisant, la pièce d'époque se présente donc, au tournant du XIX et du xx siècle, comme l'aboutissement dialectique d'un siècle de critiques muséophobiques. Mais il y a plus: au-delà de la stricte problématique de musée, l'enjeu - de nature métaphysique - consiste à vaincre le cours du temps lui-même, en opérant d'incessantes interversions entre le passé et le présent. Par l'immersion dans un habitat, les visiteurs sont incités à des expériences personnelles de remémoration et d'identification intime, quoique rêvée, à des présences humaines absentes. Par l'exposition d'usages quotidiens fondamentaux - boire, manger, dormir, travailler, etc. -, immédiatement partageables par tous, se trouvent encouragés des contacts entre la vie personnelle du spectateur et le passé étiqueté de l'exposition. Une expérience fantasmatique du passé présent en résulte, liée, dans la mise en scène de l'habitat, à la concaténation de l'espace actuel éprouvé par le corps du visiteur et du temps révolu que se représente son esprit: alors que le concept de période, en tant que tel, tend à spatialiser le temps sur un mode abstrait, la period room rêve de temporaliser l'espace sur un mode vécu. Cette incorporation de l'historicité à l'habitat rappelle alors le «domicile idéal » que Charles Baudelaire, dans une vision mi-utopique mi-addictive, identifie à un «rêvoir » où «[s'entassent] les formes anciennes» (Baudelaire 1976: 290) 11. On songe aussi à «l'historicisme narcotique» dont Walter Benjamin accusait le XIX siècle (1993: 408) et dont lui-même fut la victime consentante lorsque, après avoir consommé du haschisch, il s'est cru dans «une structure où seules des figures de cire peuvent habiter»: "Je peux faire une foule de choses avec, d'un point de vue plastique», poursuit-il; «Je peux y lire toute l'histoire du monde. Je vois dans l'espace pourquoi je collectionne des images de colportage. Je peux tout voir dans la pièce. » (Ibid. : 235)

Fantasmagorie de l'intérieur, rêve hypermnésique, narcose historiciste: autant d'expressions aptes à caractériser un programme de revitalisation imaginaire du temps historique, dans ces capsules faussement pénétrables dont la nature utopique a eu tôt fait de s'imposer à la pensée critique. Rien de plus facile, en effet, que d'identifier et de démonter les contradictions internes de cette haute promesse historico-métaphysique qui, dans la pièce d'époque, semble condamnée à se renverser en désastre, une fois dissipée la première ivresse. Contradictions dans le rapport au temps aussi bien que dans le rapport à l'espace qui, finalement, impliquent des contradictions fondamentales dans le rapport à la vie même, à travers une expérience clivée de l'intérieur habité.

\section{Défaillance du temps}

D'un côté, on l'a vu, se trouve visée une sorte d'hyper-temporalité, tissant ensemble miraculeusement le passé et le présent: spectacle d'un passé flambant neuf, sans accrocs ni poussières, parfait en sa stabilité muette que semblent devoir épargner à jamais les ravages, comme on dit, du flux temporel, «une capsule hermétiquement close», écrit Trevor Keeble, 
«intouchée par le passage du temps» (2006: 2) ${ }^{12}$. C'est-à-dire, aussi bien, le plus précieux des remèdes, en un moment de l'histoire des conceptions du temps, au cœur de la modernité industrielle, que Koselleck a décrit comme celui où «[l]a temporalisation [...] pénétrait en profondeur la vie quotidienne" (1990: 294). Alors que le cours du temps se vit désormais, jusque dans les simples actes de l'existence, sur le mode dramatique de la rupture et de l'instabilité, ces espaces de temps rêvé («Zeit-raum » en tant que "Zeittraum ", espace-temps en tant que rêve de temps, selon le jeu de mot de Benjamin [1993: 406]), sagement juxtaposés dans leurs clôtures respectives, soigneusement archivés et stratifiés pour l'éternité, devraient se donner comme une consolation, mieux même, comme une réparation des déchirures de la conscience temporelle et réancrer le vécu dans un ordre ontologique stable. C'est à quoi aspiraient, entre autres, les salles de l'«American Wing » du Metropolitan Museum of Art, lors de leur ouverture en 1924, réinventant salle après salle une histoire idéale, nette et admirablement propre de l'Amérique, au moment précis où l'afflux des étrangers provoquait l'inquiétude, conduisait à l'instauration de quotas d'immigration et incitait plus généralement au renfermement identitaire.

Sauf que cette hyper-temporalité théorique, prétendument parfaite et réconciliatrice, se trouve vidée de sa substance par l'éradication concrète des actes de la vie. Alors que tout intérieur vécu est fondé sur l'accumulation et le feuilletage temporels, associant des objets d'âges divers inégalement usés par les pratiques quotidiennes, l'intérieur d'époque aspire à une pureté historique et formelle qui ne se peut rejoindre qu'au prix de l'inauthenticité. Plus exactement: l'authenticité au regard de l'histoire périodisée est incompatible avec l'authenticité au regard de la temporalité vécue. La première exige des objets emblématiques et intacts, clairs supports de différences ordonnées entre les époques; la seconde demanderait au contraire d'affronter le désordre essentiel de la vie, qui ne se soucie pas d'identifier ni de séparer les strates de temps mais au contraire les mêle et les confond dans les meubles et objets d'usage. Plus l'intérieur s'attache à la restitution d'une époque, moins il est fidèle à l'évocation de ces pratiques vivantes désordonnées. De sorte que, loin d'harmoniser, comme promis, temps historique et temps vécu, la pièce d'époque est comme vouée à réagir contre l'impureté historique fondamentale de l'habitat, contre les adultérations, rafistolages, raboutages de toutes sortes, qui incarnent l'inexorable brouillage des catégories temporelles dans les pratiques d'habitation, et qui préexistent nécessairement à la muséification. Plus même: tout se passe comme si une sorte de mauvais génie poussait les musées à se colleter plus souvent que de raison à des pièces d'époque incertaine, dont l'ultime déménagement a été précédé par de multiples réaménagements ou déplacements antérieurs, pour différents propriétaires et à différents usages, produisant d'innombrables transformations aussi bien des meubles que des décors muraux (John Harris cite à cet égard le cas record des "sept ou huit vies" successives de la «French Gold Room " de style Louis XIII, passée par Paris, Moscou, Florence, Venise et Los Angeles avant d'atterrir au Nelson Atkins Museum de Kansas City en 1942 [Harris 2007: 184-185]).

Pour gommer autant que possible ces fâcheux défauts laissés par la vie et pour satisfaire à leurs exigences d'exactitude chronologique et de prestige visuel, les musées ont mis en œuvre, comme on sait, d'innombrables procédures: soit par la suppression d'éléments présents à l'origine mais jugés insuffisamment datables ou présentables, soit par l'addition d'éléments
12. "Spectators looking on the period room understand it only as a hermetically sealed capsule, uncontaminated by the passage of time." 
13. La pièce avait été acquise par le musée en 1922 auprès du collectionneur et marchand Charles Woolsey Lyon, qui l'avait lui-même acquise en 1921 après la destruction de la maison d'origine à Springfield.

14. Je traduis.

"For period rooms are like orphaned children; they are removed from their places and associations of origin, and placed in an alien environment. Inevitably, much of their history is lost. These rooms can reveal some of their history to the informed examiner, but on most subjects they are mute. Therefore, curators are put to the task of constructing a history for them - part fact, part fable, part informed interpretation. " étrangers à l'habitat d'origine, mais perçus comme plus représentatifs de l'époque ou plus brillants visuellement. Parfois même, les accommodements, les demi-mesures vont jusqu'à la manipulation pure et simple: le passé parfait de la pièce d'époque ne se pare alors des atours de la pureté chronologique qu'à travers une mise en scène biaisée, que contaminent en fait des intentions idéologiques modernes. Parmi d'autres exemples, Hillary Murtha a montré comment l'intérieur d'un menuisier de Springfield au XVIII ${ }^{\mathrm{e}}$ siècle, la «Reuben Bliss Bedchamber» du Brooklyn Museum of Art, en dépit de ses origines incertaines et de son état initial mal documenté, a été transformée en emblème soi-disant exact de la sévère prospérité de l'Amérique coloniale, dont la présentation muséale a évolué au gré des fantasmes contemporains, du «surameublement» («over-furnishing») luxueux, dans les années $1930{ }^{13}$ (Murtha 2005: 210), à une simplicité influencée par la culture «seventies», dans les années 1970 (fig. 5a, b, c et d):

Car les pièces d'époque sont comme des enfants orphelins; elles sont arrachées à leurs lieux et à leurs contextes d'origine, et placées dans un environnement étranger. Inévitablement, beaucoup de leur histoire s'y perd. Elles peuvent en révéler des fragments au chercheur averti, mais sur la plupart des sujets elles restent muettes. C'est pourquoi les conservateurs ont pour mission de leur construire une histoire - mi-fait mi-fable, avec une part d'interprétation avertie ${ }^{\mathbf{1 4}}$. (Ibid. : 205)

Au total, toutes les dimensions du temps se trouvent déréalisées. Le passé proposé est dès l'abord vidé de sa substance vécue: les apprêts factices de la pièce d'époque désignent des actions concrètes dont on sent immédiatement qu'elles n'ont jamais eu lieu. Tout autant, le présent est privé de sa présence, puisque les procédures actuelles de transformation en vue d'une présentation muséale demeurent inexplicites - conservateurs et installateurs déployant en général une énergie virtuose pour masquer leurs interventions. Quant au futur, il est doublement bloqué: non seulement le champ des possibles offerts à l'imagination du visiteur est restreint par la prédétermination au passé des actions qu'évoque la pièce - cette salle à manger offre le spectacle du repas et de rien d'autre, cette chambre à coucher celui du repos et de rien d'autre, etc. -, mais de surcroît, un interdit généralisé pèse sur l'accomplissement de ces actions que la pièce impose au regard: dans un monde où conservation et contemplation sont seules de rigueur, l'action réelle est évidemment taboue, sans quoi la capsule perdrait sa vertu magique. De sorte que, concrètement, la table est mise pour un repas qui n'aura jamais été pris, les bûches préparées pour un feu qui n'aura jamais flambé, toujours sous l'emprise d'un futur antérieur conjugué au négatif. «Rien n'aura eu lieu que le lieu, excepté, peut-être, une constellation »: la phrase mallarméenne d' «Un coup de dé jamais n'abolira le hasard » pourrait faire figure de devise mélancolique de la pièce d'époque.

Mélancolique est le mot, si l'on suit les analyses du psychanalyste et phénoménologue Ludwig Binswanger, selon lequel la mélancolie, dans l'ego, se caractérise par un dysfonctionnement des structures fondamentales de la temporalité, lorsque - si l'on suit le commentaire de la pensée de Binswanger par Jean Starobinski - «les actes constitutifs transcendantaux mêlent inextricablement, jusqu'à l'annulation de toute possibilité de mouvement, la rétention 

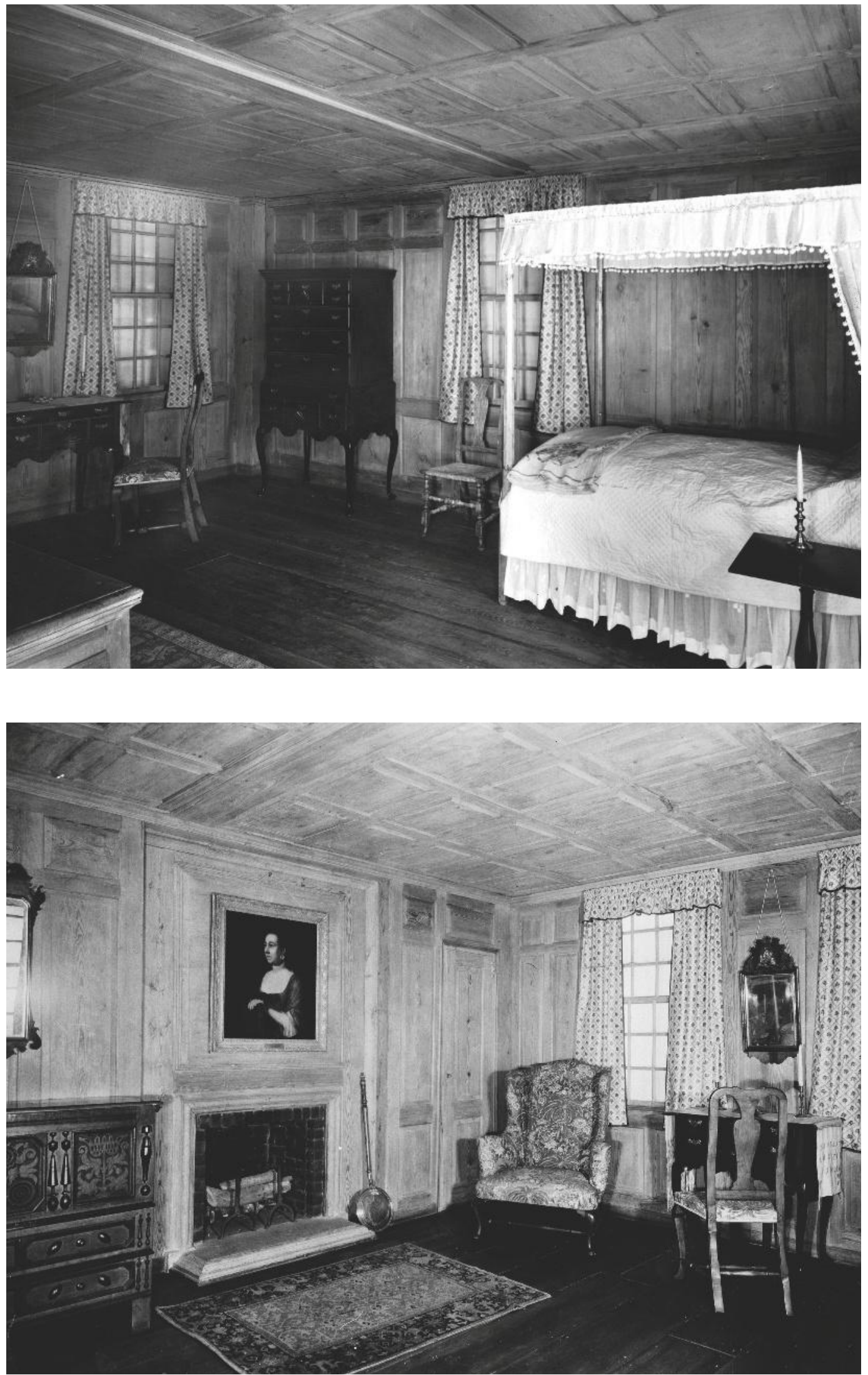
of Art, photographiée en 1929.

Brooklyn Museum of Art. Achetée grâce aux fonds donnés par le Samuel E. Haslett Estate Fund et le Alfred T. White Fund, 22.1936.

(C) Brooklyn Museum, New York. 


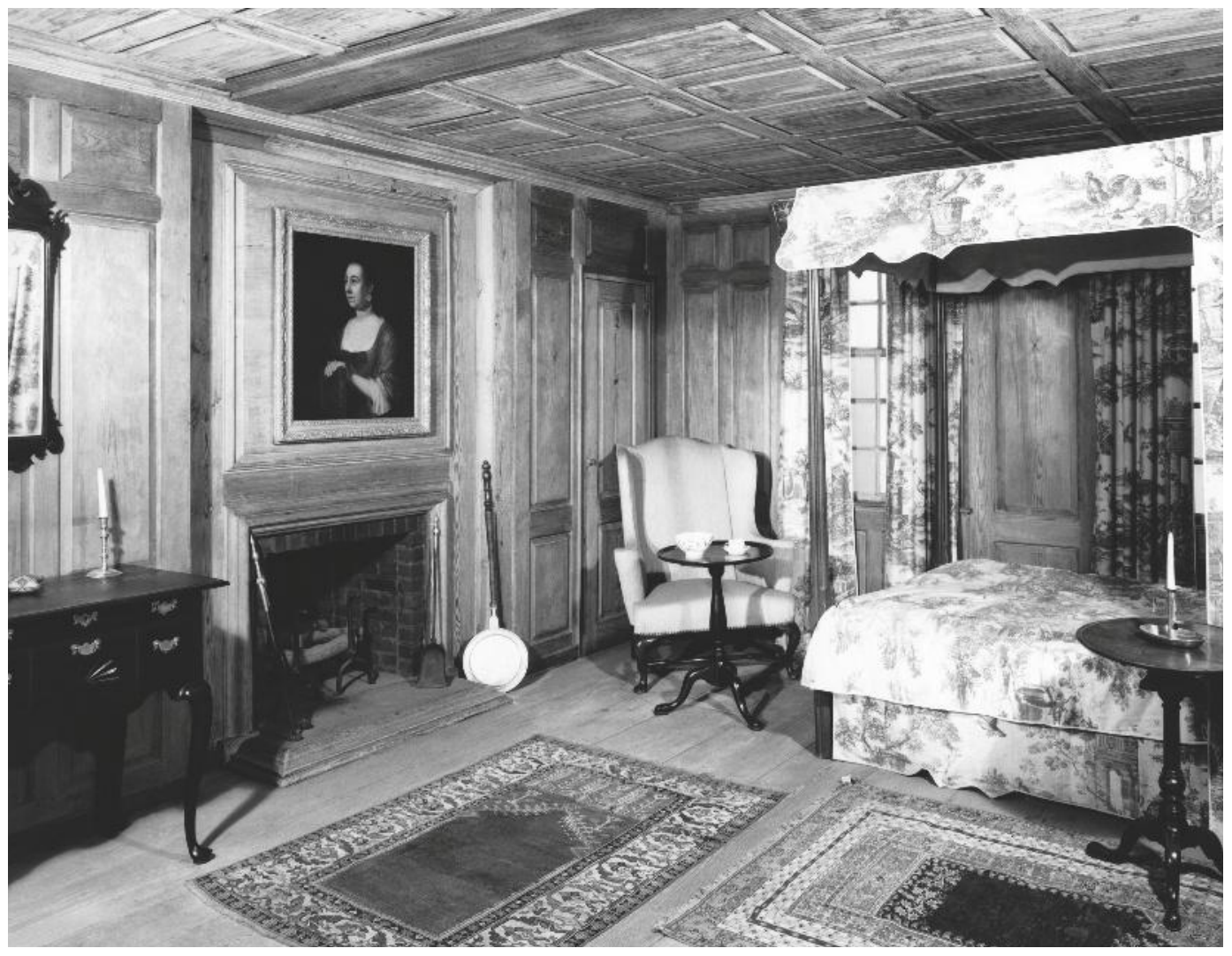

fig. $5 \mathrm{c}$

Chambre à coucher de la Reuben Bliss House (Springfield, Massachussets, vers 1754), réinstallée au Brooklyn Museum of Art, photographiée en 1945. Brooklyn Museum of Art. Achetée grâce aux fonds donnés par le Samuel E. Haslett Estate Fund et le Alfred T. White Fund, 22.1936.

(c) Brooklyn Museum, New York

\section{à droite}

fig. $5 \mathrm{~d}$

Chambre à coucher de la Reuben Bliss House (Springfield, Massachussets, vers 1754), réinstallée au Brooklyn Museum of Art, état actuel.

Brooklyn Museum of Art. Achetée grâce aux fonds donnés par le Samuel E. Haslett Estate Fund et le Alfred T. White Fund, 22.1936.

(c) Brooklyn Museum, New York.

(retentio) du passé et la protention (protensio) vers un futur hypothéqué par le regret» (Starobinski 2012: 178). Dans la mélancolie, la «protention est infiltrée de moments rétentifs » (Binswanger 1987 [1960]: 37) et réciproquement, ce qui produit une désorganisation générale du moi et un effondrement de sa capacité à s'établir dans l'expérience du présent ou «présentation». Par une sorte de cercle vicieux, les constituants transcendantaux de la temporalité s'interpénètrent et se détruisent au sein de la conscience ainsi affectée:

Là où la présentation ne peut pas s'appuyer sur des intentions rétentives et là où elle ne peut pas projeter sur des possibilités protentives ouvertes, donc là où l'unité de la synthèse des actes constitutifs de l'objectivité temporelle est «relâchée» et où des fils isolés se détachent de la «trame» de cette synthèse et s'entremêlent, là se modifie le «style» tout entier de l'expérience et partant aussi le «style» de la réalité du monde. (Ibid. : 51)

Le «style» qui en résulte, pour Binswanger, est celui de la perte: "L'expérience continuera de se dérouler selon le même style, à savoir le style de la perte, précisément en tant "qu'expérience mélancolique" ou expérience de la réalité des mélancoliques. » (Ibid.) Perte du moment de la présentation, 


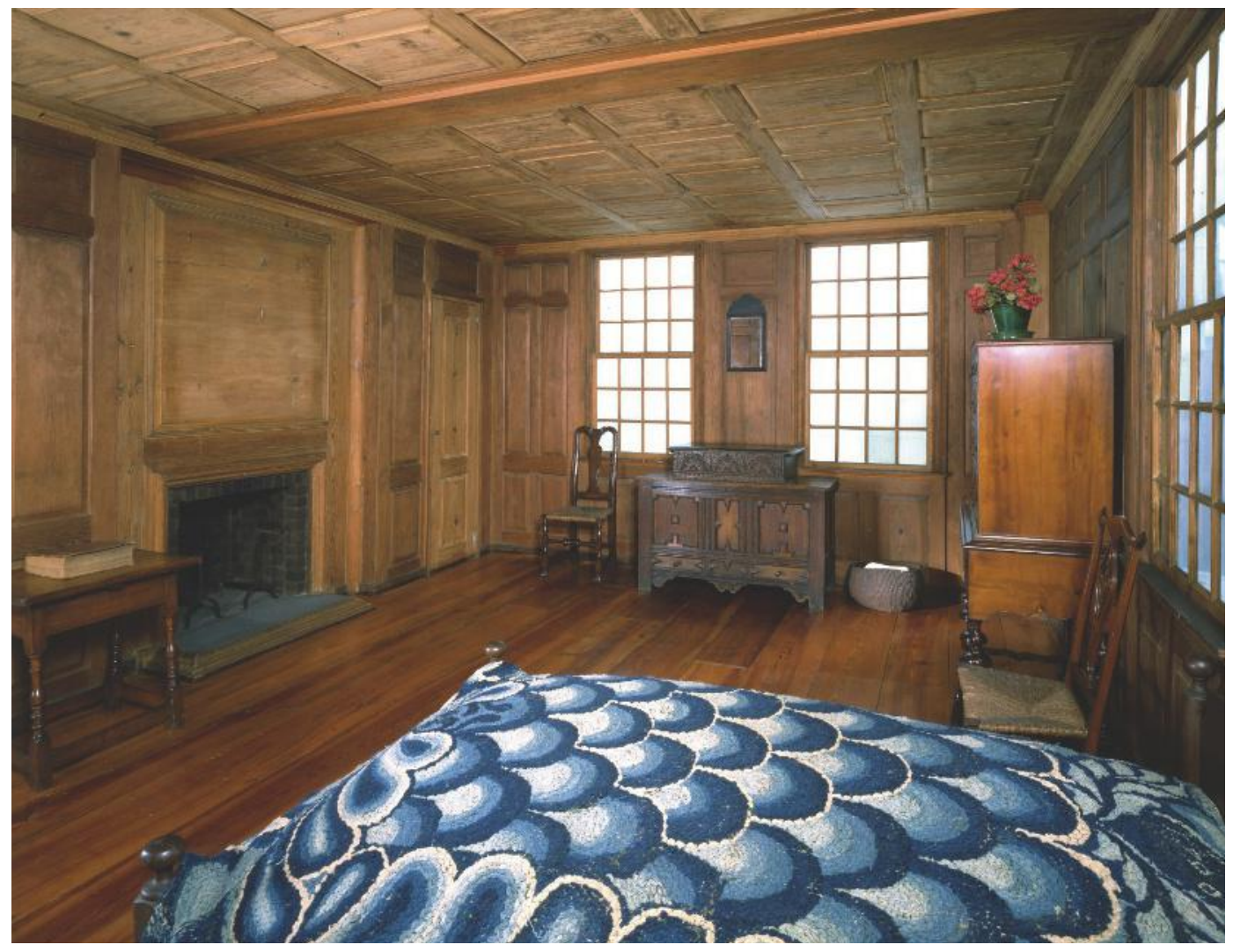

annulé par l'emmêlement de la rétention et de la protention, et perte, surtout, du moment de la protention vers le futur, c'est-à-dire de l'imprévisible élan de la vie, au profit d'un fantasme permanent de déjà-accompli:

Ce que nous remarquons en premier lieu quand nous considérons le caractère de perte de la protention mélancolique, c'est sa perte de plénitude et de puissance, ou plus précisément sa limitation à une seule ou à un petit nombre de possibilités. Mais «l'impuissance» de ces possibilités s'accompagne d'une puissance d'autant plus grande, puissance qui s'exprime dans une évidence aussi inébranlable que celle propre d'ordinaire à la seule rétention. [...] Le mélancolique considère en règle la perte, non pas comme devant advenir, mais comme déjà réalisée. (Ibid. : 49-50)

La pièce d'époque est l'espace par excellence de déploiement et de formulation de cette temporalité originellement «défaillante» (ibid. : 115) ${ }^{\mathbf{1 5}}$, effondrée sur elle-même, propre à la mélancolie. Idéalement une promesse, elle est réellement un chaos; idéalement une essence encapsulée, réellement une figure de la fuite et de la perte: le lieu des fantômes, là où, pour une conscience que paralysent insidieusement les spectres, la communion entre les vivants et les morts échoue et s'altère en brouillage anxiogène, comme se brouille également la différence entre le passé et le futur. Ce n'est donc pas
15. La « défaillance de la constitution temporelle». 
sans raison que le premier exemple, hors contexte muséal, que donne le muséologue Raymond Montpetit pour qualifier les «images de la muséographie analogique », est funèbre: «Les intérieurs d'époque ont existé hors des musées, bien avant qu'on en ait une notion claire. Les familles qui ferment la chambre d'un parent décédé et la conservent longtemps dans cet état en font, avec le temps, un "intérieur d'époque". " (Montpetit 1996: 70)

\section{La victoire des choses}

Ce qui se joue dans le rapport au temps se joue identiquement dans le rapport à l'espace. Là aussi, l'idéal promis tend à se renverser en catastrophe, au moment de l'expérience concrète. L'idéal: une perméabilité fluide entre l'intérieur et l'extérieur, l'immixtion merveilleuse du public venu du dehors dans un espace privé. L'installation des spectateurs de plain-pied, en continuité avec la scène, leur mise en situation de quasi-participation, tout cela relève d'une volonté d'«immersion», pour reprendre une catégorie de Montpetit, qui «vise la simulation intégrale» et «plonge les visiteurs physiquement au centre de la scène reproduite, afin qu'ils éprouvent concrètement l'environnement» (ibid. : 92). Ainsi devrait s'accomplir la victoire sur les artifices d'une esthétique théâtrale propre au musée, au profit d'une forme de vérité affective totale. Sauf qu'aussitôt se dresse la contradiction: l'inévitable «mise à distance» - cordon, vitre ou sonnerie électronique -, comme on l'appelle dans le jargon muséal, contraint les cheminements et interdit le contact physique avec les objets; les barrières prolifèrent. À la valeur d'usage de l'espace, au premier degré, est substituée, au second degré, l'exposition de cette valeur d'usage et cette exposition même produit comme fatalement la destruction de son objet. Le principe de la mise en exposition force le spectateur à adopter un comportement exactement contraire à celui auquel invitent par ailleurs l'espace et les objets: il lui faut contempler au lieu d'empoigner; rester debout au lieu de s'asseoir ou de se coucher; ne pas manger au lieu de festoyer; passer son chemin de salle en salle au lieu d'habiter. Plutôt qu'accueilli dans l'habitat exposé, il reste prisonnier, comme disait Benjamin, du «sortilège des seuils» («Schwellenzauber») (Benjamin 1993: 232), relégué aux marges d'un inutilisable espace d'exposition.

Suivant un mécanisme de double contrainte, deux postulations contraires - l'inclusion et l'exclusion, la perméabilité et l'imperméabilité, l'usage et le non-usage, l'invitation à habiter et l'interdiction de le faire se superposent et font éprouver avec une acuité accrue l'incompatibilité douloureuse entre les deux valeurs d'usage et d'exposition. D'où, pour la conscience écartelée entre ces deux pôles, un réflexe d'angoisse que le sentiment de claustrophobie incarne de manière privilégiée. L'étroitesse des zones d'accès et l'emboîtement des espaces les uns dans les autres n'en sont pas la cause première mais ils en renforcent l'effet: murs dans des murs, cubes dans des cubes, fenêtres sans dehors éclairées de faux jours, tout contribue à resserrer l'étau d'une impression d'enfermement.

Au bout du compte, une poétique initiale d'incorporation de l'intérieur objectif de la pièce à l'intériorité subjective du visiteur se renverse donc en son contraire: ce n'est pas l'espace qui s'incorpore au moi intime et l'enrichit; c'est le moi intime qui se désagrège et se dissout dans un espace d'autant plus tyrannique qu'il est impraticable. Le «domicile idéal », rêvé par Baudelaire 
après Edgar Allan Poe, dans l'introduction à la Philosophie de l'ameublement (Baudelaire 1976: 290), aurait dû être une extension du moi dans l'espace, une innervation de l'espace intérieur par la vie intérieure, faisant des objets, par l'usage, de purs prolongements de l'être-au-monde subjectif. Mais la spectacularisation de l'intérieur habité implique son maintien à distance - à la fois réelle et symbolique. Et sous le signe de l'angoisse, face au spectacle d'un intérieur interdit, qu'on ne nous présente que pour nous en écarter, la cohérence intérieure du moi vacille et s'effondre. Ce n'est pas l'intérieur qui, idéalement, vibre de la vie du moi; c'est le moi qui, mélancoliquement, est happé par le pouvoir mortifère de l'intérieur, selon un processus d'inversion qui, chez Baudelaire, conduit du rêve opiomane de la Philosophie de l'ameublement au cauchemar de Spleen II: «Je suis un vieux boudoir plein de roses fanées, / Où gît tout un fouillis de modes surannées" - écho au «cimetière abhorré de la lune" auquel le poète vient aussi de s'identifier (Baudelaire 1975 [1857]: 73). Plus l'intérieur s'exhibe en tant que capsule temporelle, plus se vérifie son pouvoir de fracturation de l'intériorité.

À la tension majeure entre pénétrabilité et impénétrabilité, productrice de claustrophobie, se superpose une tension entre stabilité et instabilité, qui aiguise l'effet d'anxiété par une forme de désorientation. L'idée prétendument rassurante d'une capsule de temps spatialisé, exhaustivement offerte à la vision panoramique dans le champ d'un espace fermé, est minée par l'inquiétante étrangeté d'un monde déplacé, immeuble transformé en meuble, maison mouvante qui suggère la soumission de ce qu'il y a de plus stable, de plus enraciné - la demeure - à la loi implacable de l'impermanence et du déracinement. À l'endroit précis où s'annonçait l'installation d'un ordre solide, sourd et s'aggrave le sentiment du désordre. L'interdiction de tout contact n'apparaît plus alors que comme un fragile garde-fou, ne s'opposant qu'en surface, dérisoirement, à une menace profonde et constante de désagrégation.

Autant dire que la pièce d'époque, dans sa structure intrinsèquement contradictoire (donc tragique), se dresse contre la montée d'un sentiment du néant dont elle a pourtant méticuleusement orchestré la venue par sa fictionnalisation de la vie. Face au néant, la pièce d'époque se remplit; elle est pleine: pleine de choses - «over-furnished» - et pleine de significations. Chacune des choses qui la remplissent, agencées comme dans un kaléidoscope (cette autre invention fantasmatique du $\mathrm{XIX}^{\mathrm{e}}$ siècle), y est sur-signifiante, ne serait-ce que par le redoublement de son sens, au titre de la documentation des pratiques quotidiennes et au titre du prestige visuel. Mais ce plein désigne un vide, ou plutôt une absence abyssale, celle des corps vivants, alors même que les objets réclament leur présence active pour rentrer dans l'usage et donc dans la vie. Or les seules présences charnelles sont celles des visiteurs, observateurs passifs d'un théâtre vide, rejetés dans les marges au point qu'il arrive - comme à la Provost Skene's House d'Aberdeen, en Écosse (Meldrum 1958-59) - qu'on agence les miroirs de telle manière que nous ne puissions jamais nous y apercevoir. Le contraire, en somme, d'un tableau vivant, qui forme en quelque sorte le pendant de la pièce d'époque dans une même fascination pour les points de basculement fantasmatique entre les images et la vie: dans le tableau vivant, les individus résistent, in fine, à leur devenirobjet et l'image un instant fixée à laquelle ils collaborent ne tarde pas à se dissoudre à nouveau dans le flux de la vie (Vouilloux 2015; Halimi 2011); dans la pièce d'époque, à l'inverse, les objets résistent, in fine, à leur devenirvivant, et la vie possible qui aurait pu les animer se fige irrémédiablement en image, inaugurant le règne des fantômes.

\section{double page suivante}

fig. 6

Ronan Jim Sévellec, Le champs clos des ablutions païennes, 2016-2017.

Avec l'aimable autorisation de l'artiste et de la galerie Antonine Catzéflis, Paris. Photo Joël Laiter. 


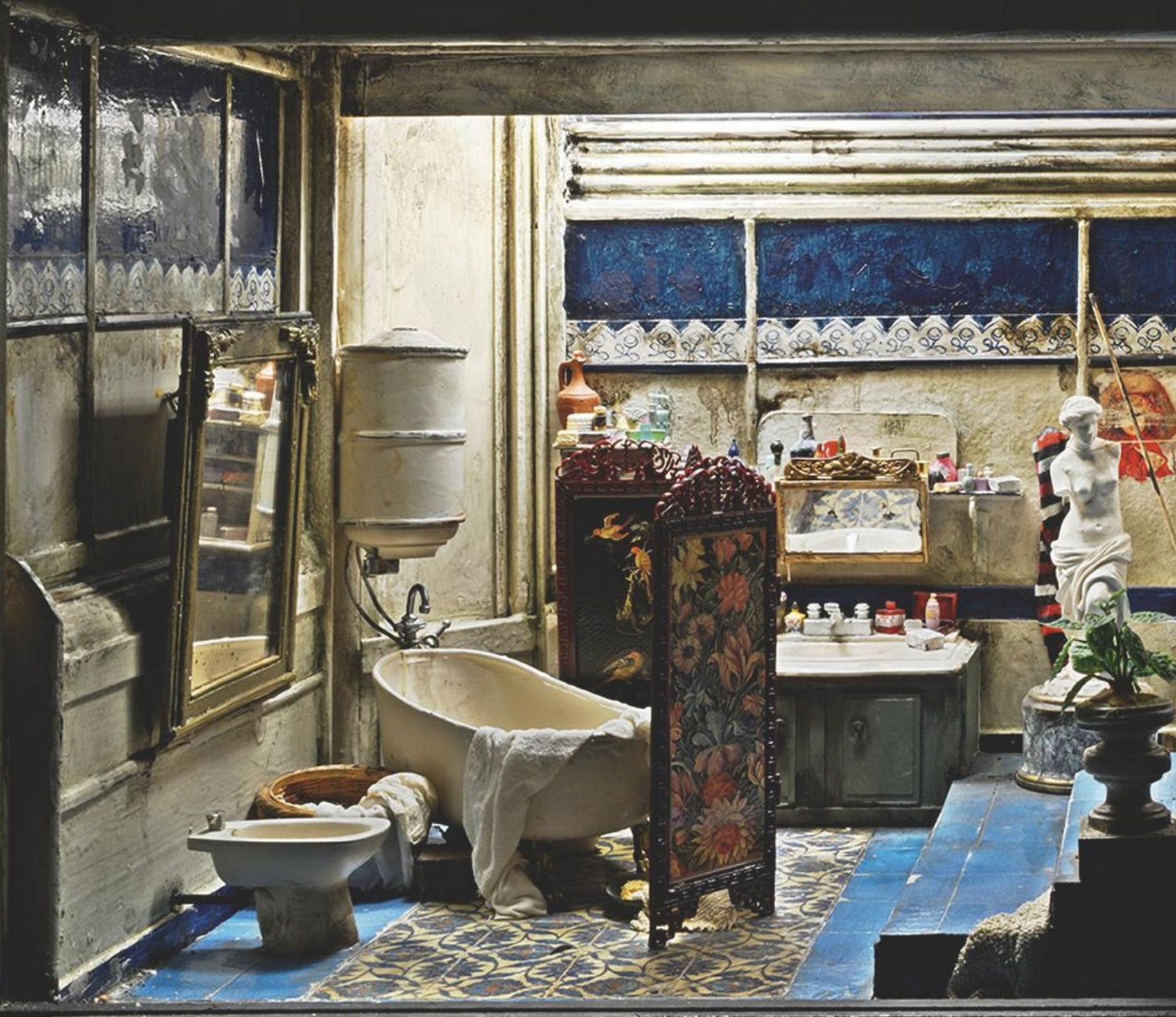




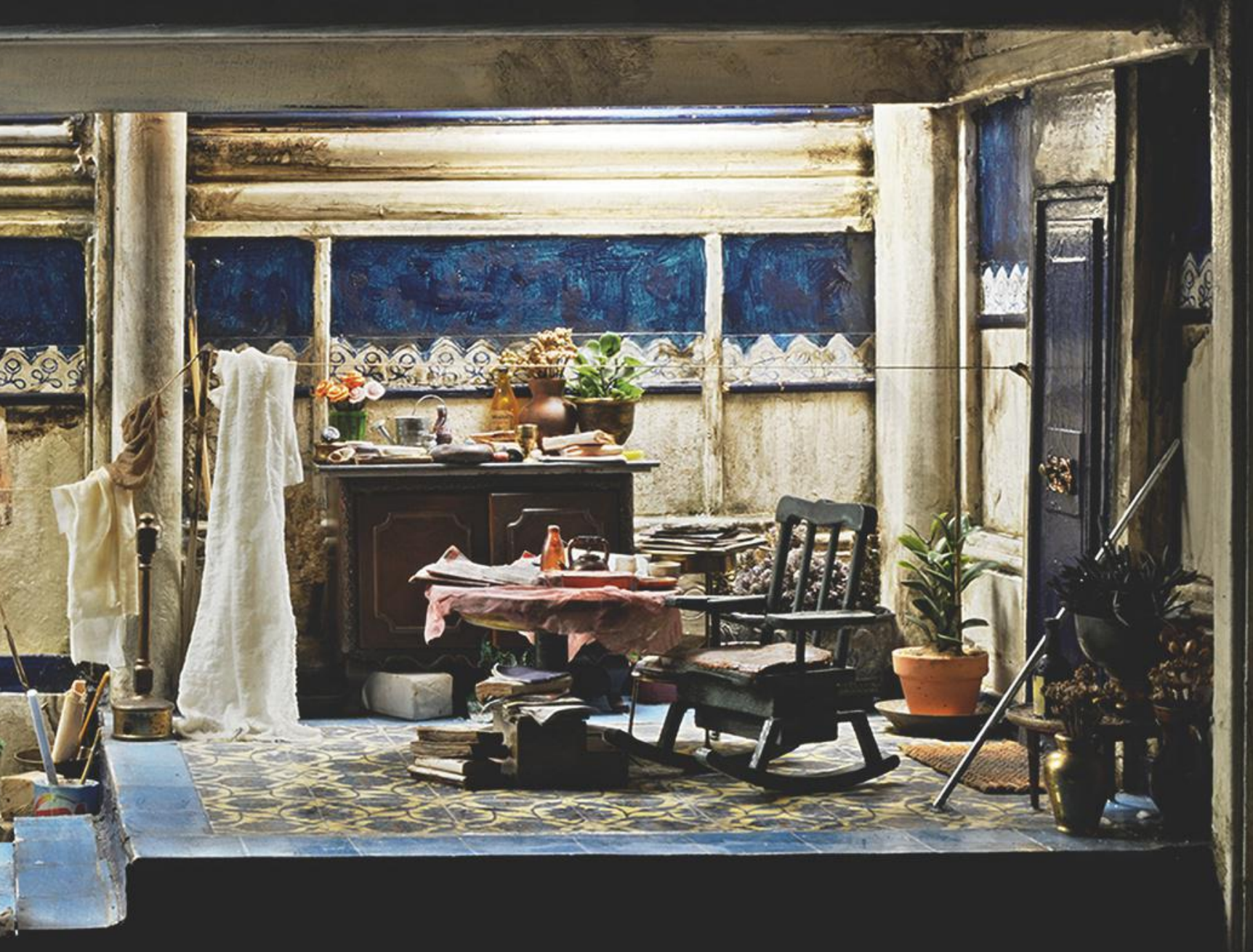




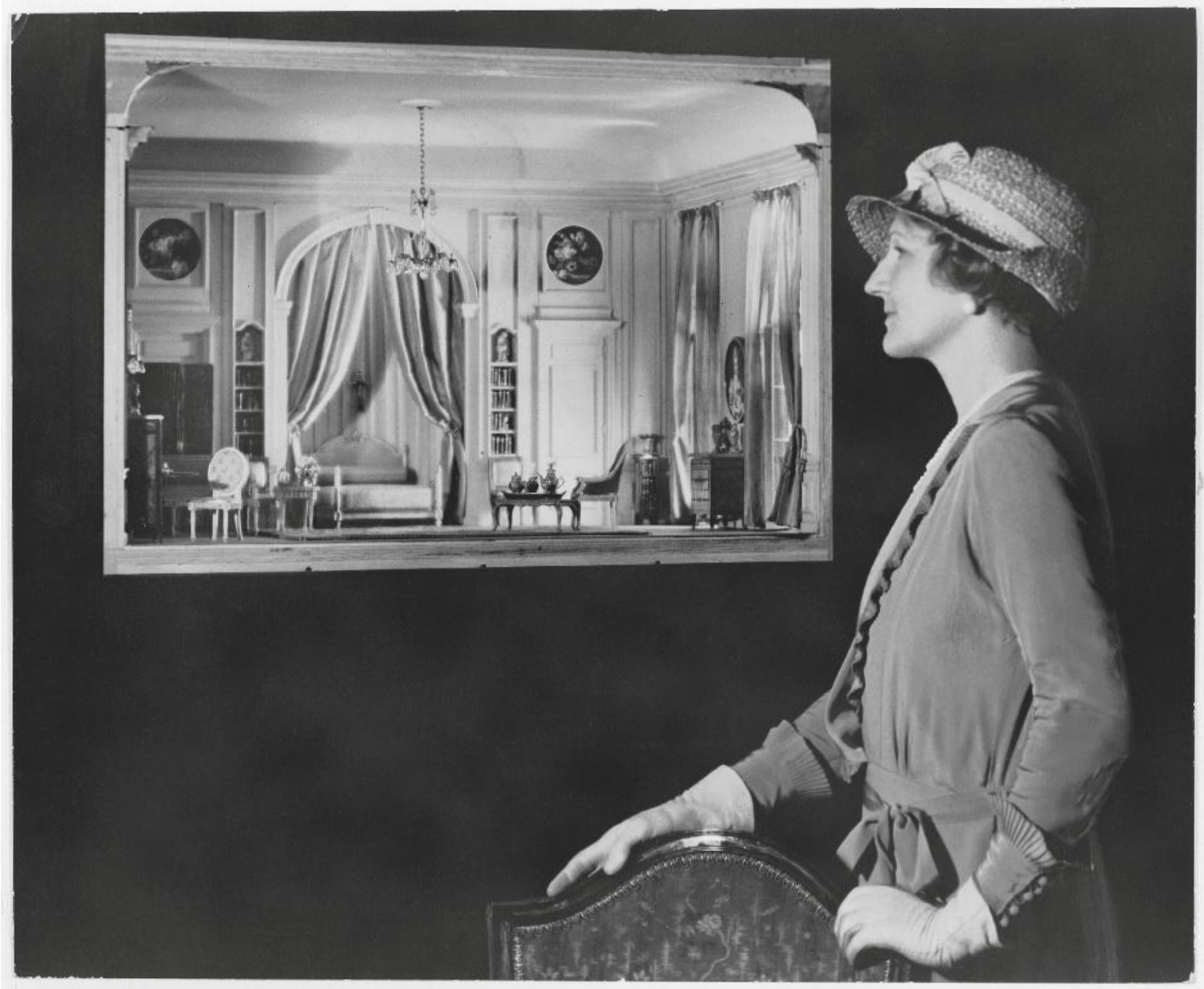

fig. 7

Narcissa Niblack

(Mrs. James Ward Thorne)

posant devant sa chambre

à coucher miniature

d'époque Louis XVI,

exposition «Chicago

Historical Society », 1932,

ou «Century of Progress

Exhibition ", 1933.

Avec l'aimable autorisation

de The Art Institute

of Chicago. 
Sur ce plan matériel (espace et objets compris) comme sur le plan de la temporalité, cette sensation d'une victoire des choses sur les corps, des images sur les actes, de l'objectivité matérielle sur l'affectivité vivante est, encore une fois, un parfait symptôme de la mélancolie - lorsque, comme l'écrit Starobinski à propos des Spleens des Fleurs du mal, «[l']inventaire des choses surpasse numériquement celui que nous pourrions faire des indices affectifs » (Starobinski 2012: 447). Ces choses livrées à elles-mêmes en même temps que chargées de missions signifiantes acquièrent un poids de présence hallucinatoire qui procède directement de leur impossible statut de substituts. Le spectateur, assailli par leur plénitude factice et par une vacuité réelle, se trouve confronté à l'injonction mentale de donner vie à ce qui est sans vie. Mais face au «théâtre des objets ", selon l'expression de Manuel Charpy (2010), l'absence d'autres vivants engendre insidieusement, pour celui qui pénètre dans cet espace en attente d'une impossible action, un sentiment d'absence à soi - angoisse de désubstantiation individuelle qui peut inciter à la fuite hors de l'air raréfié des salles de musée mais qui, aussi bien, par la jouissance paradoxale qui l'accompagne, peut provoquer une forme de fixation contemplative, saisie par le spectacle étrange du vide. C'est cette sorte de contemplation figée qu'a, par exemple, identifiée Bruce Hatton Boyer devant les pièces miniatures conçues par Narcissa Niblack Thorne dans l'entre-deux-guerres et exposées depuis 1954 à l'Art Institute de Chicago (fig. 7): “C'est précisément l'absence de figures qui rend les Thorne Rooms de l'Art Institute si fascinantes. [...] Nous sommes requis de fournir l'ingrédient crucial qui manque - la vie - et c'est ce qui fait que nous restons rivés si longtemps en face de nos pièces préférées ${ }^{\mathbf{1 6}}$. " (Boyer 1983: 24)

\section{Mélancolie d'un présent sans présence}

Ce réseau de contradictions qui affecte structurellement les pièces d'époque explique sans doute l'inquiétude qu'elles n'ont pas tardé à provoquer au sein même des musées, de la part de conservateurs qui, après un premier moment d'enthousiasme - relativement bref, à tout prendre, et marqué en particulier par un développement extraordinaire aux États-Unis dans l'entre-deux-guerres $\mathbf{1 7}$ - ont majoritairement œuvré à leur remise en cause, aboutissant à de nombreux démantèlements et à des "désacquisitions ", suivant le terme chastement consacré pour désigner une sortie définitive hors des collections.

La première cause de défaveur est venue des doutes sur leur authenticité historique, au fur et à mesure que l'expertise en matière d'arts décoratifs s'est aiguisée dans les équipes de conservation (d'où, parmi bien d'autres exemples, la vente de la "Lawrence Room» par le musée de Boston dès 193018 [Harris 2007: 151-152], ou le retour de la «Gwydir Castle Room» du Metropolitan Museum à son château d'origine, en $1996{ }^{19}$ [ibid.: 163-164]). Parallèlement, la montée en puissance d'une muséologie analytique de l'objet singulier, soigneusement isolé, et de la forme pure a conduit à voir dans ces environnements plus ou moins factices des repoussoirs, représentants à la fois naïfs et coupables d'une muséologie analogique (ce fut le cas pour une partie des "Thorne Miniature Rooms », que l'Art Institute de Chicago a revendues à l'entreprise IBM dans les années 1940, peu de temps après les avoir reçues en donation, les jugeant sans doute plus proches de la maison de poupée fantaisie que de l'œuvre d'art 20 [Boyer 1983: 15]). Plus récemment,
16. Je traduis.

"It is the very absence of figures which makes the Art Institute Thorne Rooms so compelling. [...] We must furnish the crucial missing ingredient - life which is why we can stand riveted in front of our favorite rooms for so long. "

17. Voir Duncan 2005 : 227-231. "Period rooms and period displays had become the blockbuster phenomena of the interwar period in the United States. " (Duncan 2005

228). L'ouverture de period rooms par George Francis Dow à l'Essex Institute de Salem, Massachusetts, en 1907, est regardée comme un point de départ, et l'inauguration de

l'«American Wing» du Metropolitan Museum of Art de New York, en 1924 sous la houlette de R.T.H. Halsey, comme un couronnement.

18. Ensemble composite de fragments d'ébénisterie flamands et anglais du $\mathrm{XV} \mathrm{I}^{\mathrm{e}}$ siècle, artificiellement assemblés à Londres dans des officines de marchands, la «Lawrence Room » avait été donnée au Boston Museum of Fine Arts par la veuve du colone Timothy Lawrence en 1876 et est considérée par John Harris comme la première pièce historique européenne importée en tant que telle aux Etats-Unis.

19. La pièce fut vendue par les propriétaires de Gwydir Castle, au Pays de Galles, en 1921, à l'antiquaire new-yorkais French \& Co, achetée par William Randolph Hearst, donnée par la Hearst Foundation au Metropolitan Museum of Art en 1956.

Jamais exposée car reconnue comme inauthentique, elle a finalement été rendue au château en 1996.
20. Après le don de ses pièces miniatures par Mrs. Thorne à l'Art Institute en 1940, cette institution a vendu les trente premières réalisations à IBM Corporation, qui les a présentées dans des expositions itinérantes, puis dispersées: redécouvertes en 1960 dans la vitrine d'un magasin new-yorkais par le fils de Mrs. Thorne, elles furent réacquises, grâce au soutien financier d'IBM, rendues à Mrs. Thorne, restaurées et partiellement offertes au Phoenix Art Museum et à la Dulin Gallery of Art de Knox Ville, Tennessee. 
21. Pour un autre exemple de critique politique de la pièce d'époque, à propos des pièces créées au Metropolitan Museum of Art en 1929 au sein de l'exposition «The Architect and the Industrial Arts ", voir Wilson 2005.

22. Sur l'appartenance de R.T.H. Halsey à I'«Americanization Movement " et sur la tonalité réactionnaire et xénophobe de son discours lors de l'inauguration de l'«American Wing ", voir Kaplan 1982 : 49-50.

23. Bryant 2009: 73-84, à l'occasion de la réouverture, en 2007 au Metropolitan Museum of Art de New York, des "Wrightsman Galleries for French Decorative Arts ", dont la restauration sous forme de pièces d'époque a été confiée au décorateu d'opéra et de mode Patrick Kinmonth.

24. Je traduis.

"The effect is one of deep melancholy and irony at the same time. [...] A labyrinthine wandering through a deserted, ruined town called the past [...]. Soane revealed himself as a proto-Poststructuralist. ” enfin, des critiques idéologiques ont dénoncé leur orientation souvent conservatrice, sexiste et nostalgiquement arc-boutée sur des mythes identitaires (nationaux ou régionaux): bref, fondamentalement essentialiste et donc propagatrice de clichés culturels, comme l'a exemplairement montré Wendy Kaplan (1982) à propos de R.T.H. Halsey ${ }^{21}$, le très républicain designer et conservateur des period rooms américaines du Metropolitan Museum of Art en 1924, dont il voulait faire un lieu pédagogique pour la diffusion, disait-il, de «nos principes américains perdus» («our lost American principles ») 22 (Duncan 2005: 229). Tout militait donc, dans les musées en quête de modernité, pour la marginalisation de la pièce d'époque, revendue, renvoyée en réserve ou maintenue à titre de simple curiosité - promise, en somme, au sort de tant d'autres phénomènes expositionnaires du XIX ${ }^{\mathrm{e}}$ siècle: panoramas, pantomimes, exhibitions de monstres, théâtres d'ombres et autres divertissements défunts ou moribonds, qui se sont d'autant plus facilement éteints qu'ils laissaient la place aux fantasmagories mondiales du cinématographe.

Pourtant, tout bien considéré, ce qui frappe plus encore que sa décadence programmée est la résistance, envers et contre tout, du dispositif de la pièce d'époque dont, dans les musées, ni l'érudition positiviste de l'objet historique singulier ni la valorisation moderniste de la forme pure ni même la pensée de la déconstruction ou l'éthique post-coloniale n'ont jamais pu avoir complètement raison. Plus même, on observe, depuis la fin du siècle dernier, un certain retour en grâce ${ }^{\mathbf{2 3}}$, stimulé par la remise en cause ironique des utopies modernistes et la montée en puissance d'un historicisme ludique. C'est ce qui explique, par exemple, l'intérêt renouvelé pour un personnage comme l'architecte et collectionneur londonien John Soane, décrit par Wolfgang Ernst comme un «proto-post-structuraliste» des années 1810-1820, dont la maison-musée, où s'accumulent compulsivement les fragments d'antiques, apparaît emblématique de la pensée historique sous-jacente à toute pièce d'époque: un «vagabondage labyrinthique à travers une ville désertée et ruinée appelée le passé», provoquant chez le spectateur des «effets simultanés de profonde mélancolie et d'ironie 24 » (Ernst 1993: 485-486). Dès 1812, au moment précis où il décide de transformer sa demeure en musée, Soane se décrit lui-même comme un archéologue du futur découvrant les ruines de son propre musée et se demandant quelle avait bien pu être la fonction de ce bâtiment (Soane 2015 [1812]) : étrange jouissance à prévoir ainsi l'effondrement programmé de l'intention d'encapsulage muséal qui présidait au même moment à la transformation de sa maison en mausolée sursaturé de fragments d'antiques, au centre souterrain duquel allait trôner, à partir de 1824, le sarcophage vide du pharaon Seti ler, ténébreusement éclairé de l'intérieur par des lueurs de bougie (fig. 9). Dans un double mouvement contradictoire, la capsule temporelle archi-pleine et son contraire, la fuite du temps et la fatalité du vide, coagulent dans un système de signes dont l'intentionnalité inséparablement constructrice et déconstructrice, militante et ironique, suscite immanquablement la fascination post-moderne.

Ce retour désabusé mais délicieux - délicieux parce que désabusé? sur soi peut aller jusqu'au narcissisme muséal lorsque des institutions d'aujourd'hui entreprennent de proposer à la délectation des visiteurs l'histoire de leur propre muséographie, en sanctuarisant d'anciennes mises en scène pourtant présentées comme surannées et en faisant partager au public le plaisir trouble d'une adhésion minée par la désillusion (pensons, au-delà des pièces d'époque occidentales stricto sensu, au maintien actuel et à la restauration 
Des pièces d'époque aux capsules temporelles. Temps historique et temps vécu dans l'expérience esthétique. Par Rémi Labrusse

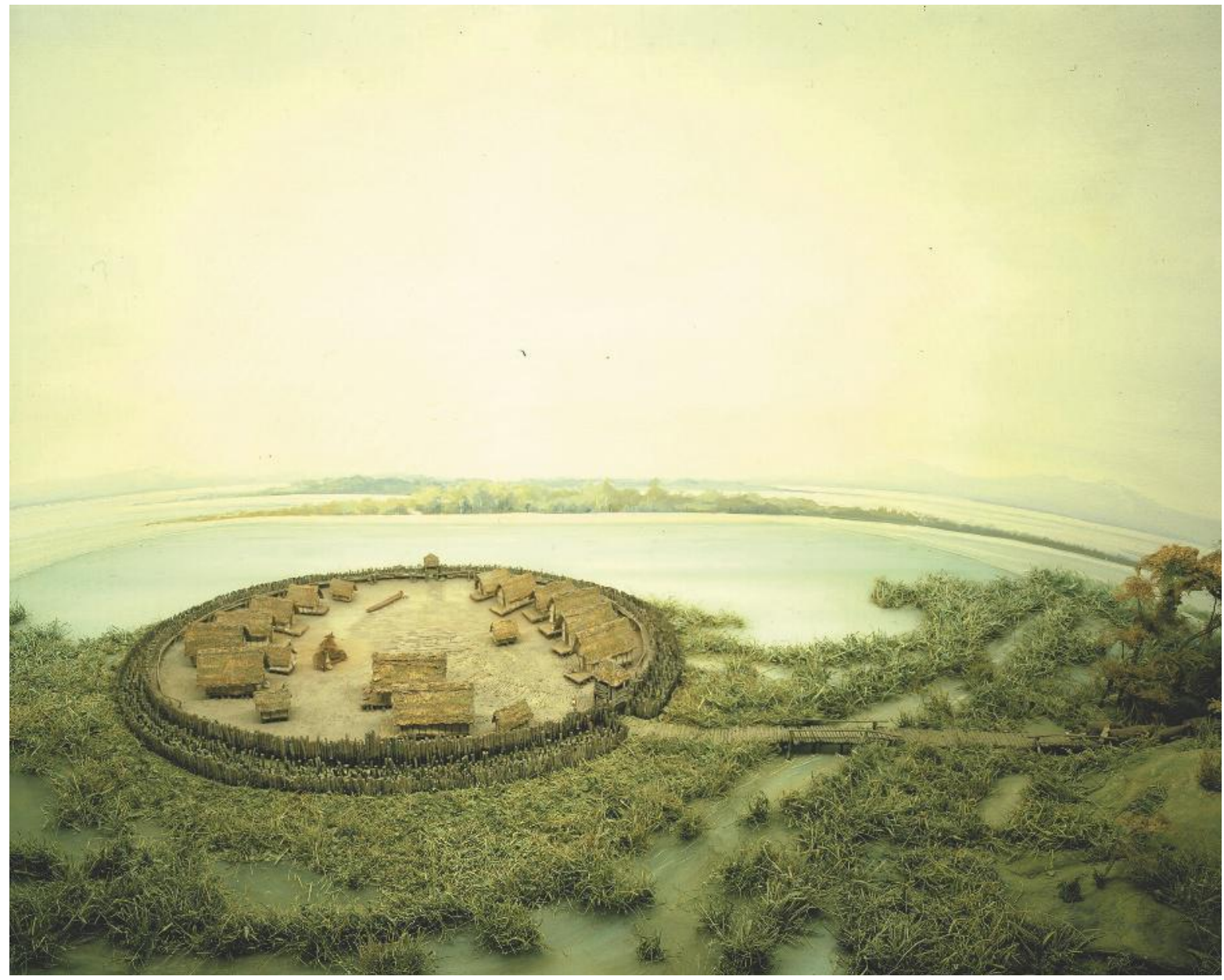

fig. 8

Diorama de la reconstruction

d'un village sur pilotis sur

la rive du lac de Contance,

Berlin. Réalisé d'après

les découvertes et

analyses archéologiques,

vers 3000 av J.-C.

(c) BPK, Berlin, Dist.

RMN-Grand Palais /

Jürgen Liepe. 


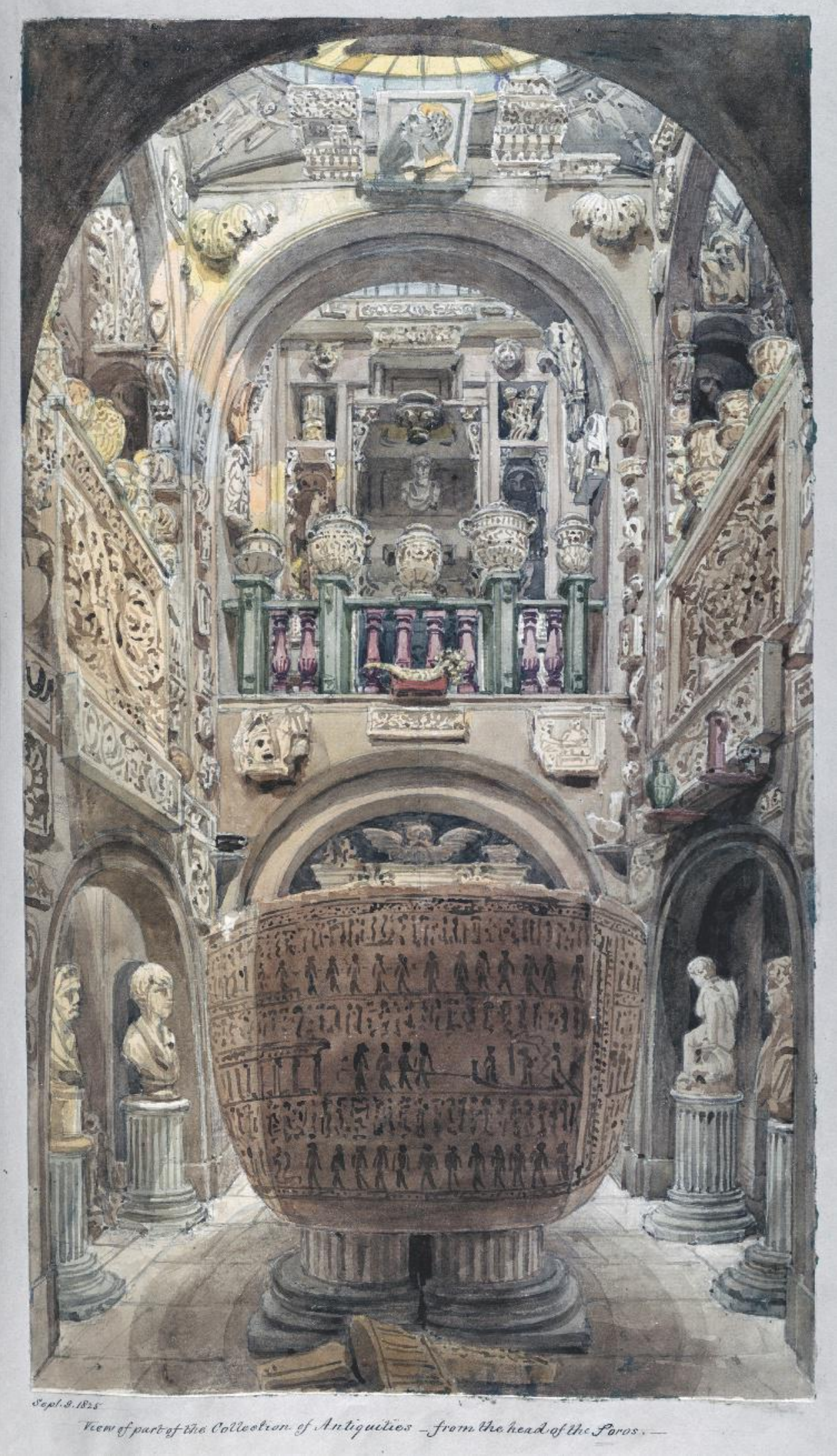


continue des dioramas ethnographiques, à fortes connotations genrées et raciales, de l'American Museum of Natural History de New York et à leurs mises en scène spectrales dans la série photographique des Dioramas d'Hiroshi Sugimoto à partir de 1976 [fig. 10]). Mais si la pensée de la déconstruction et l'esthétique du kitsch ont conféré aux usages distancés de la pièce d'époque une actualité accrue, ce n'est qu'en se mettant au diapason d'une tendance de fond, originaire: l'association entre pièce d'époque et mélancolie historiciste.

Comme le jeu désillusionné dont elle est aujourd'hui la source, la mélancolie, on l'a vu, fut originellement à l'œuvre dans l'expérience de la pièce d'époque: le dispositif lui-même, aussi bien temporel que spatial, en rassemblait pragmatiquement les conditions de possibilité. Faisons maintenant I'hypothèse qu'il en constitue aussi, poétiquement, l'allégorie en trois dimensions: la mélancolie, autrement dit, n'est pas seulement produite, elle est également réfléchie par le dispositif de la pièce d'époque. Là, dans cette structure si singulière, le sujet est confronté à la figure de ce qu'il éprouve au moment même où il l'éprouve, de sorte que les moyens lui sont donnés non pas de diagnostiquer analytiquement, comme du dehors, les raisons de ses affects, mais de creuser réflexivement dans l'affect même, d'opérer une sorte de mise à distance intérieure en médiatisant ce qu'il éprouve sans jamais cesser de l'éprouver immédiatement: ce que permet la figuration allégorique. Plutôt qu'une capsule temporelle univoque ou que la mise en scène également univoque de sa vanité, la pièce d'époque se donne en son fond comme le lieu d'une pensée critique de la pulsion d'encapsulage temporel.

Dans l'épreuve mélancolique au premier degré, les structures temporelles s'emmêlent, la vie extérieure se transforme en théâtre, le tempo subjectif intérieur se ralentit jusqu'à se figer, les indices affectifs cèdent sous le poids des objets et ces objets acquièrent un pouvoir hallucinatoire en devenant, comme l'ensemble du monde environnant, les surfaces de projection d'une perte originelle indéterminée dont «l'ombre s'abat sur le moi », pour reprendre la célèbre caractérisation freudienne de l'affect mélancolique 25 (Freud 1968 [1916]: 155-156) (par opposition au deuil, dans lequel le sujet identifie au contraire clairement l'objet de la perte et n'est pas, par conséquent, contaminé lui-même, dans ses structures constitutives, par l'épreuve de cette perte). Dans la pièce d'époque, une extrême ambition de départ - la revivification permanente de l'épaisseur du temps passé par la médiation d'objets historicisés - s'effondre, révélant les apories principielles qui l'affectent. Tout un présent sans présence conduit non pas à regretter un objet ou un moment donné, dont on pourrait alors faire le deuil, mais à souffrir mélancoliquement d'une perte inqualifiable, celle du temps vivant comme tel, de la présence précisément. Soumis à un réseau de doubles contraintes, incapable de désigner concrètement ce qu'il a perdu mais certain de l'évidence de la perte (qu'accuse encore la vaine profusion des choses assemblées), le sujet risque d'en être réduit à se retourner contre soi, à s'auto-accuser en se voyant comme la source de son insituable malaise, bref à se vivre lui-même comme désintégré, désagrégé du tissu de la vie: inguérissable expérience de désubjectivation, perte de soi qui constitue, en propre, la mélancolie.

À l'égard de l'espace où se produit cette épreuve peuvent correspondre deux attitudes: un processus d'attachement morbide (ou cyniquement ludique, ce qui va de pair); et un réflexe de fuite (né de cette «fatigue muséale ", de cette horreur du musée-cimetière dont d'innombrables visiteurs,
25. "L'investissement d'objet [dans le processus mélancolique] s'avéra peu résistant, il fut supprimé, mais la libido libre ne fut pas déplacée sur un autre objet, elle fut retirée dans le moi. Mais là elle ne fut pas utilisée de façon quelconque : elle servit à établir une identification du moi avec l'objet abandonné. L'ombre de l'objet tomba ainsi sur le moi [...] [Der Schatten des Objekts fiel so auf das Ich]."

\section{ci-contre}

fig. 9

Joseph Michael Gandy, Crypte sous la coupole du Soane's Museum, avec le sarcophage de Seti ${ }^{e r}$, encre et aquarelle sur papier, 9 septembre 1825

() The Trustees of Sir John Soane's Museum. 

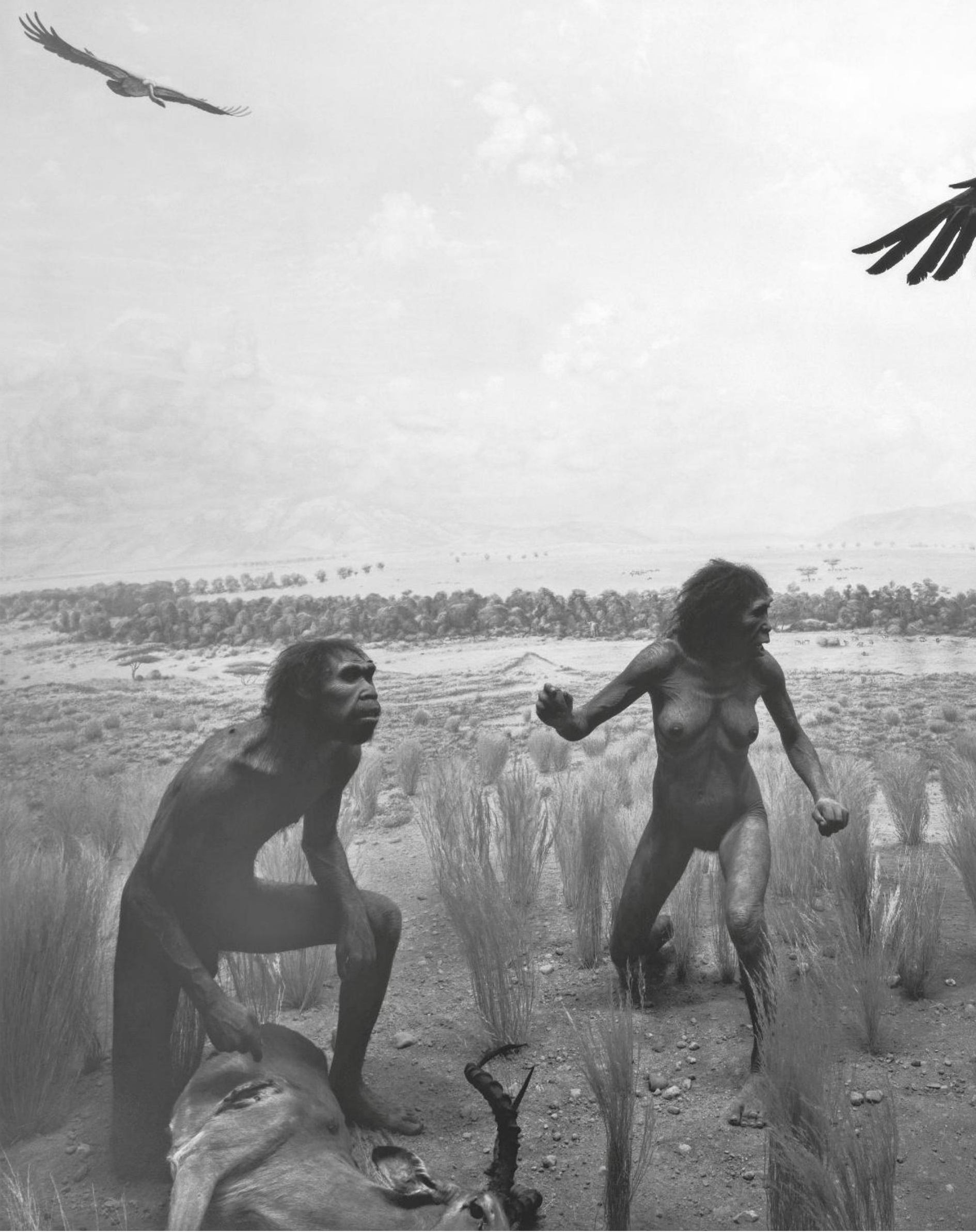
même les mieux intentionnés, ont un jour ou l'autre subi les attaques, en général plus vives que jamais lorsqu'il s'agit d'aborder une enfilade de pièces d'époque - souvent peu fréquentées -, dans lesquelles les pas résonnent entre des rangées de meubles inaccessibles).

Si elle demeurait sur le plan de l'affect, sans être recomposée par la pensée, cette épreuve intériorisée de la perte appartiendrait strictement au registre de la mélancolie au sens freudien, lorsque le moi ignore ce qu'il a perdu et, par conséquent, le recompose en lui-même jusqu'à s'autodétruire (le sentiment diffus de culpabilité provoqué par l'ennui des musées a à voir avec cela). Mais, dans l'enceinte de la pièce d'époque, un second mouvement intervient, mouvement d'auto-compréhension qui procède de la nature allégorique du dispositif. Quelles que soient les époques mises en scène, des attributs récurrents - la désertion des êtres vivants là où tout les appelle; les faux jours immuables à travers de fausses fenêtres translucides, bloquant les accidents du dehors et le passage rythmé des heures; les bûches préparées dans les cheminées pour des feux qui ne brûlent jamais, etc. - donnent corps, si l'on peut dire, à une poétique du blocage qui se déploie obstinément de pièce en pièce et y allégorise la « désorganisation et l'enchevêtrement des mouvements rétentifs et protentifs de la structure temporelle», la «défaillance de la constitution temporelle» (Binswanger 1987: 14 et 115), c'est-à-dire le noyau même, selon Binswanger, de l'angoisse mélancolique.

Certaines grandes poétiques muséales, à l'aube de la pensée du musée moderne, ont fait culminer ces attributs allégoriques dans des figures explicites de la mort: tombeaux et cénotaphes du musée des Monuments français d'Alexandre Lenoir, vers 1800 ; sarcophage égyptien vide de la collection de Soane, vers 1825. À des degrés d'intensité divers, autrement dit, la pièce d'époque pense toujours et donne toujours à penser la mélancolie qu'elle produit. Mais dans cet acte poétique de production sensible et de mise à distance intellectuelle simultanées s'opère aussi une transformation décisive: la pièce d'époque n'apparaît plus simplement comme l'espace de déploiement d'un affect mélancolique; elle devient l'espace d'accomplissement d'un travail de deuil, à l'égard des ambitions propres à toute fabrication de capsule temporelle.

\section{L'ouverture par le deuil}

Le visiteur de la pièce d'époque n'y recueille pas que les éléments d'une histoire des formes et des usages, représentés par un complexe d'objets et d'éléments matériels de toutes sortes. II n'y vit pas seulement non plus, en posture mélancolique, l'échec d'une mise au présent du passé et, du coup, l'embrouillement paralysant de sa propre temporalité vécue. Plus en profondeur, à un niveau allégorique, oscillant entre le concept et l'image, entre l'intellection et l'empathie, il y recueille un savoir intime de sa mélancolie et se donne les moyens de l'explorer, donc de s'en désaliéner: ainsi la voie se trouve ouverte pour ce que Freud a précisément nommé un travail de deuil («Trauerarbeit»).

Ce qui, paradoxalement, vit, c'est-à-dire ce qui se produit, ce qui a lieu dans les pièces d'époque et qui explique leur insistante résistance en tant que pratique muséale, c'est ce travail de deuil, rendu possible par la mise en perspective allégorique d'une mélancolie première. Et de quoi, par un tel travail, le sujet se sépare-t-il? Du rêve de pouvoir figurer et donc contempler sur un mode historique sa propre épaisseur subjective. La pièce d'époque est l'espace 
Des pièces d'époque aux capsules temporelles. Temps historique et temps vécu dans l'expérience esthétique.

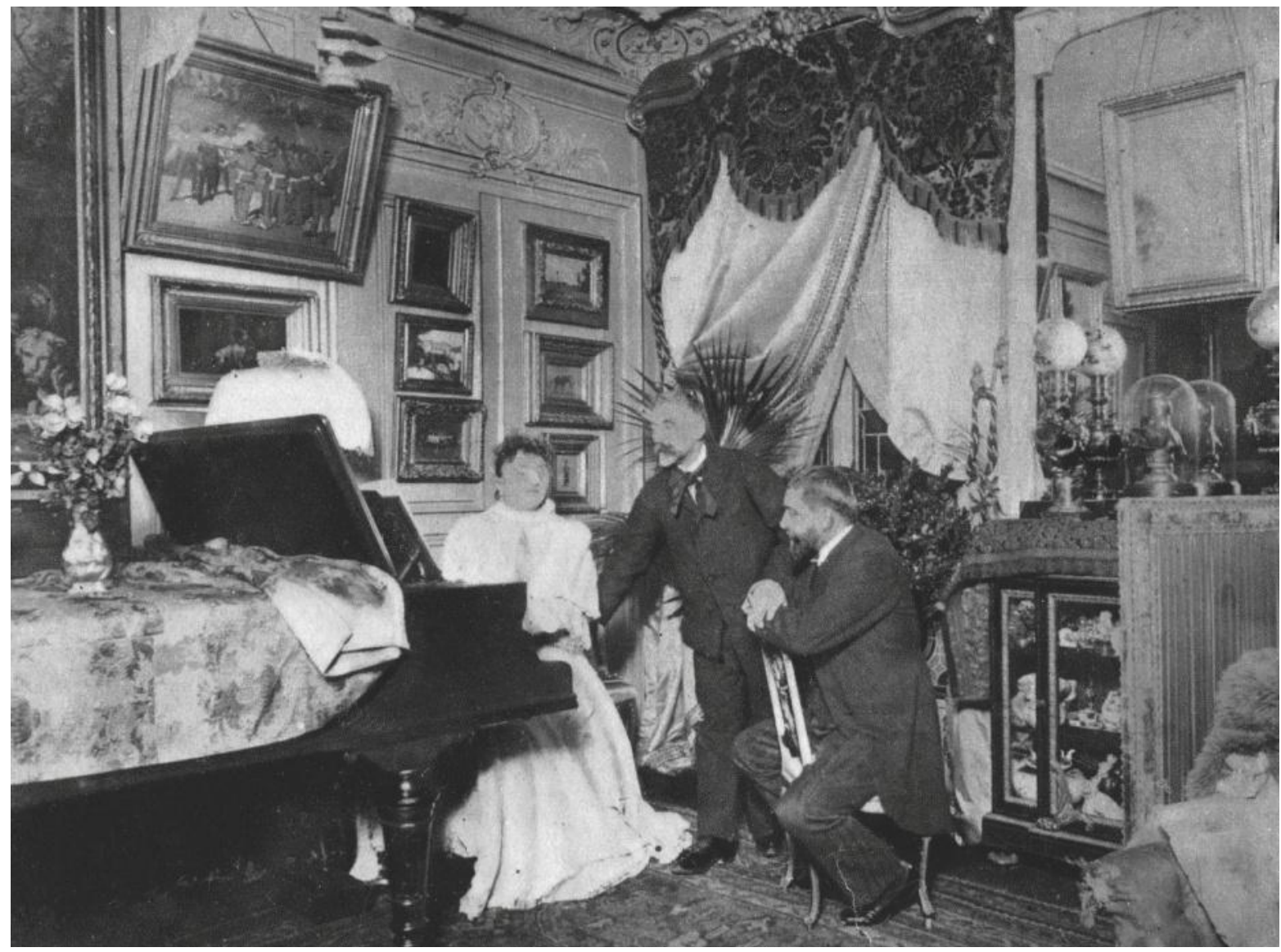

fig. 11

Paul François Arnold

Cardon, dit Dornac,

Stéphane Mallarmé,

Henri Gervex et Méry

Laurent chez Méry Laurent,

villa des Talus,

19 boulevard Lannes

à Paris, vers 1891

(c) Heritage-Images

/ The Print Collector /

akg-images. 
par excellence au sein duquel se défait l'illusion que la vie intime, l'intériorité subjective puisse être authentiquement projetée dans un dehors objectif et recueillie par l'histoire (ce qu'emblématisait l'idée de capsule). La leçon première d'historicité - voir la vie passée, sous forme parfaitement circonscrite - s'y dissout dans une méditation métaphysique seconde. L'expérience peut être fugitive et indistincte, le temps de quelques pas, d'une entrée et d'une sortie, d'un regard jeté, d'un frisson ressenti sans qu'on sache exactement s'il est de plaisir ou d'angoisse; même confuse et labile - à peine une irisation sur les eaux de la conscience -, elle n'en contient pas moins ces différentes dimensions - ou tonalités - entrelacées: I'histoire vécue ne s'y donne à voir que pour aussitôt s'effriter; les noces rêvées de l'histoire et de la vie, se dérobant, laissent la conscience désemparée, incapable de recomposer sa propre structure temporelle, encagée dans ses propres apories; et dans ce paysage mental de ruines, s'esquisse une pensée spectrale de l'inadéquation de la subjectivité vivante à tout ce que l'histoire pourrait en dire. Ajoutons que, comme dans tout travail de deuil (par opposition à la spirale entropique de la mélancolie), la souffrance engendrée par l'évidence de la perte trouve sa récompense dans une rémunération: le moi mélancolique qui s'éprouvait au départ comme déshabité, ravagé, déserté, se découvre désaliéné, constatant sa radicale hétérogénéité par rapport à une temporalité objective et périodisée. Le silence obstiné des objets à l'égard de la vie même ne débouche pas seulement sur une épreuve mélancolique du néant mais aussi et simultanément sur une intuition renouvelée de l'écart entre vie et récit figuratif.

Autrement dit: dans la pièce d'époque, la plainte mélancolique du visiteur - se percevant comme le dérisoire alter ego de choses mortes, de mannequins et de fantômes - se situe toujours au plus près de son renversement, au plus près d'une apothéose du moi se reconnaissant, selon les mots de Mallarmé à son ami Henri Cazalis, comme «ce solitaire habituel de sa propre Pureté, que n'obscurcit plus même le reflet du Temps » (Mallarmé 1995: 342). Le jeune homme, il est vrai, avait d'abord prévenu son correspondant: «Heureusement, je suis parfaitement mort» (ibid.); et c'est sans doute hanté par la conscience mélancolique de cette radicale absence à soi que le poète a été fasciné sa vie durant par l'inquiétante étrangeté des ameublements de son temps, peuplés de bibelots, se passionnant même obsessionnellement, vers 1890, pour la confection de l'intérieur claustrophobique de sa maîtresse Méry Laurent (Pety 2010: 277-283) [fig. 11]. La ligne de démarcation est donc fragile, entre l'épreuve catastrophique du néant et l'émergence glorieuse d'un sentiment anhistorique de la vie. Ou plutôt: l'une ne cesse de s'emmêler à l'autre, donnant à l'expérience muséale en général, et à celle de la pièce d'époque en particulier, sa pesanteur et son obscurité, sa richesse et sa vérité - ne fût-ce, encore une fois, que le temps d'un coup d'œil. Lorsque la mélancolie existentielle, allégorisée, parvient à s'y transformer en deuil et lorsque le travail de deuil, à son tour, parvient à y rouvrir la dimension du futur, la vérité qui surgit en creux est celle de l'irréductibilité de la subjectivité à ses conditions historiques d'apparition dans le monde. Vérité métaphysique s'il en est, dont les abîmes allégoriques de la pièce d'époque, à leur façon négative, permettent l'émergence, expliquant par là l'étrange alliance de répulsion et d'attraction que ces petits mondes crépusculaires de présences absentes ne cessent pas de susciter. 


\section{Coda}

Deux remarques adjacentes pour finir.

La première à partir d'une image: en 1929, dans le numéro 5 de sa revue Documents, George Bataille intitule Greniers, mannequins, débris et poussières une vue des réserves du Musée d'ethnographie du Trocadéro, figurant un entassement désordonné de caisses, d'objets et de mannequins dans une pièce close: contre-pièce d'époque où, derrière la scène, dans les greniers, l'ordre se renverse en désordre, la propreté en poussière, l'intégrité en débris, les humains en pantins - mais parachèvement, aussi bien, d'une émotion critique qui s'est lovée au principe même de la poétique de la pièce d'époque, chambre d'écho où résonne la perplexité de l'Occident moderne face à l'étrangeté de sa propre invention, le phénomène muséal.

Seconde remarque, à partir d'une découverte: en janvier 1995, la grotte ornée paléolithique Chauvet-Pont d'Arc a été scellée et interdite au public, avant même que sa découverte, quelques jours plus tôt, ait été officiellement annoncée. Presque aussitôt, on a préparé son avatar, une pseudo-grotte à quelques kilomètres de là, dans l'esprit de ce qui s'était déjà fait à Lascaux et à Altamira auparavant. Ce fac-similé combine aujourd'hui l'aura de l'artisanat manuel, mimétique du premier moment paléolithique (les copies peintes ont été réalisées à la main par une équipe de peintres), et les prestiges de la technologie la plus «moderne», forte de ses machines et de ses algorithmes: hyper-pièce d'époque, sanctifiée par l'onction perpétuelle d'une quasi parfaite capsule temporelle, la vraie grotte interdite - mais écroulement, aussi bien, de l'ambition ontologique à l'œuvre dans les pièces d'époque, puisqu'en l'occurrence l'authenticité l'a désertée en se repliant dans une cella d'un nouveau genre, aussi proche qu'inaccessible, et en laissant place, dans la néo-grotte, à un divertissement spectaculaire désillusionné.

Au total, le désir clivé au travail dans toute structure apparentée à une pièce d'époque apparaît ainsi comme le symptôme, plus actuel que jamais, d'une angoisse propre à la modernité, dont l'objet est la temporalité comme telle. Cet obscur désir collectif n'est pas tant celui de créer des capsules temporelles à l'écart du présent que de confronter ces artifices mélancoliques à leurs apories et de garder sauf, souterrainement, un horizon émotionnel de «présence » d'autant plus insistant qu'il demeure insaisissable.

Université Paris Nanterre remi.labrusse@u-paris10.fr
Remerciements :

Ce texte trouve son origine dans une communication au colloque Comme si...

Espaces et fictions. Autour de la period room, organisé par Pascal Griener et Odile Nouvel, avec l'assistance de Dora Precup, à l'Institut national d'histoire de l'art à Paris, les 16 et 17 novembre 2012. Je les en remercie tous trois vivement. 

18 bets

Vouilloux, Bernard

2015 Le Tableau vivant.

Phryné, l'orateur et le peintre.

Paris, Flammarion.

Wilson, Kristina

2005 «Style and Lifestyle in the

Machine Age: The Modernist Period Rooms of "The Architect and the Industrial Arts" ", Visual Resources. An International Journal of Documentation 21 (3) : 245-261. page 76 et ci-contre Joseph Michael Gandy, Crypte sous la coupole du Soane's Museum, avec le sarcophage

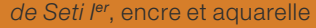
sur papier, 9 septembre 1825 (détail) @ The Trustees of Sir John Soane's Museum.

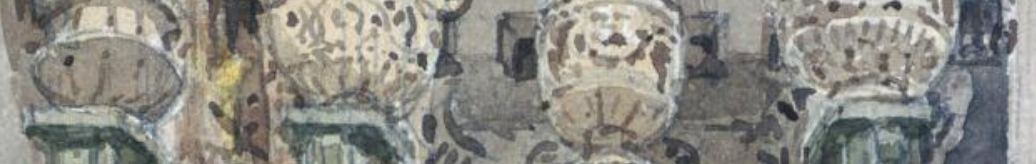
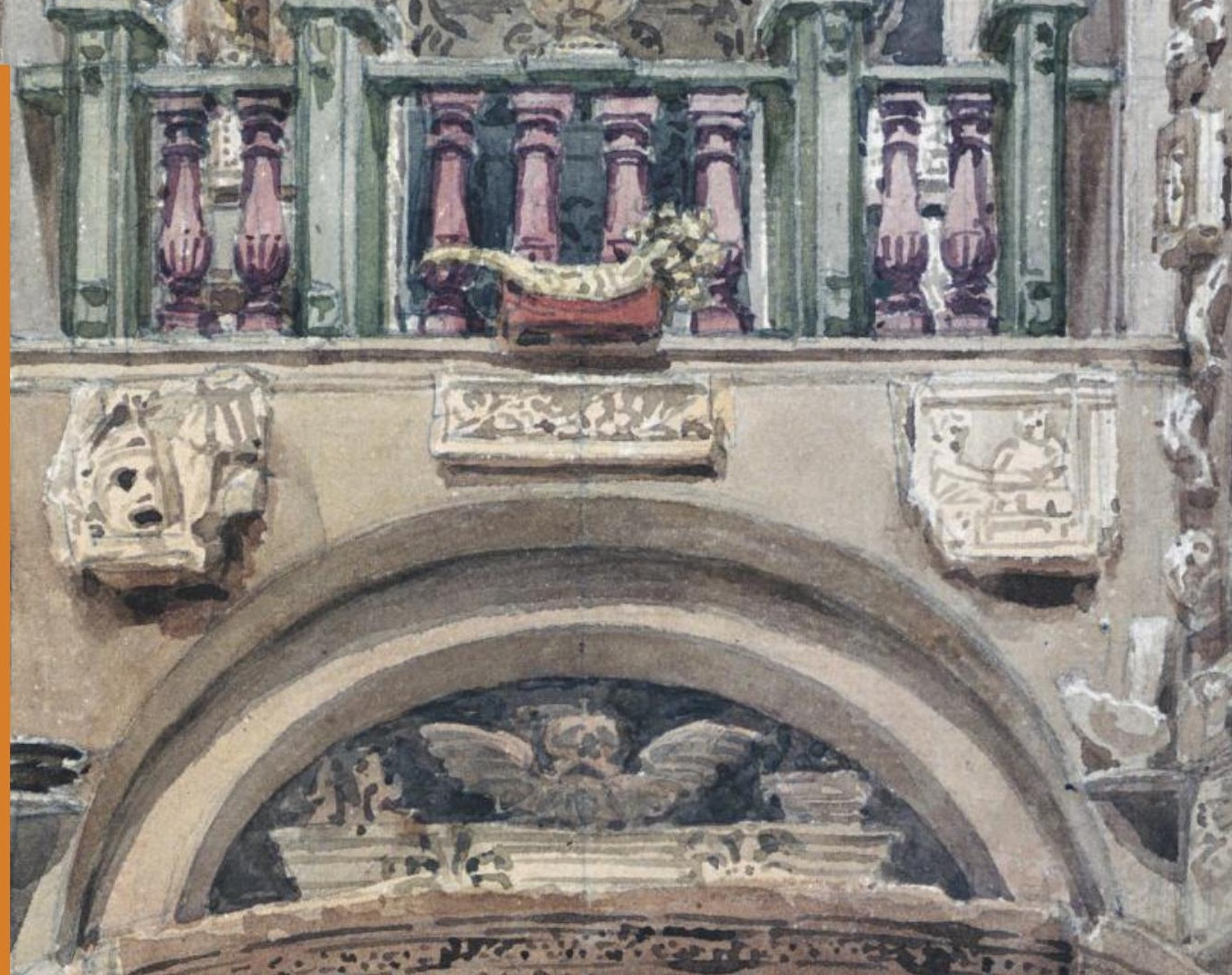
(1)

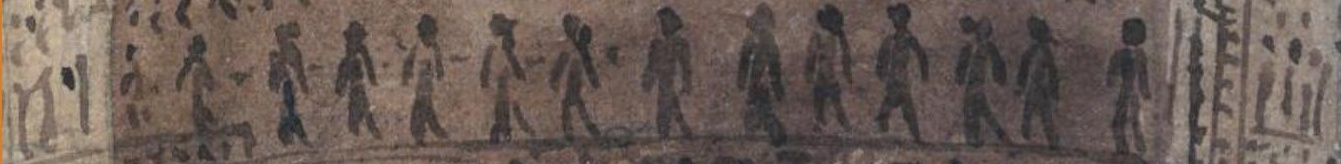
2007.

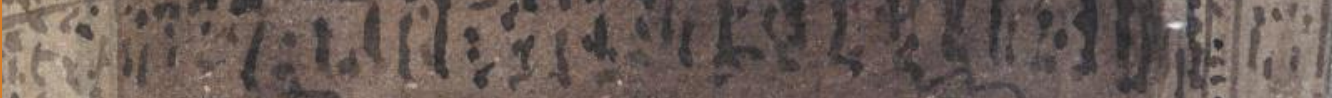

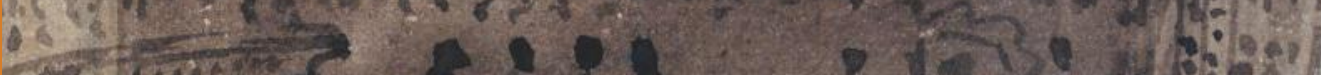

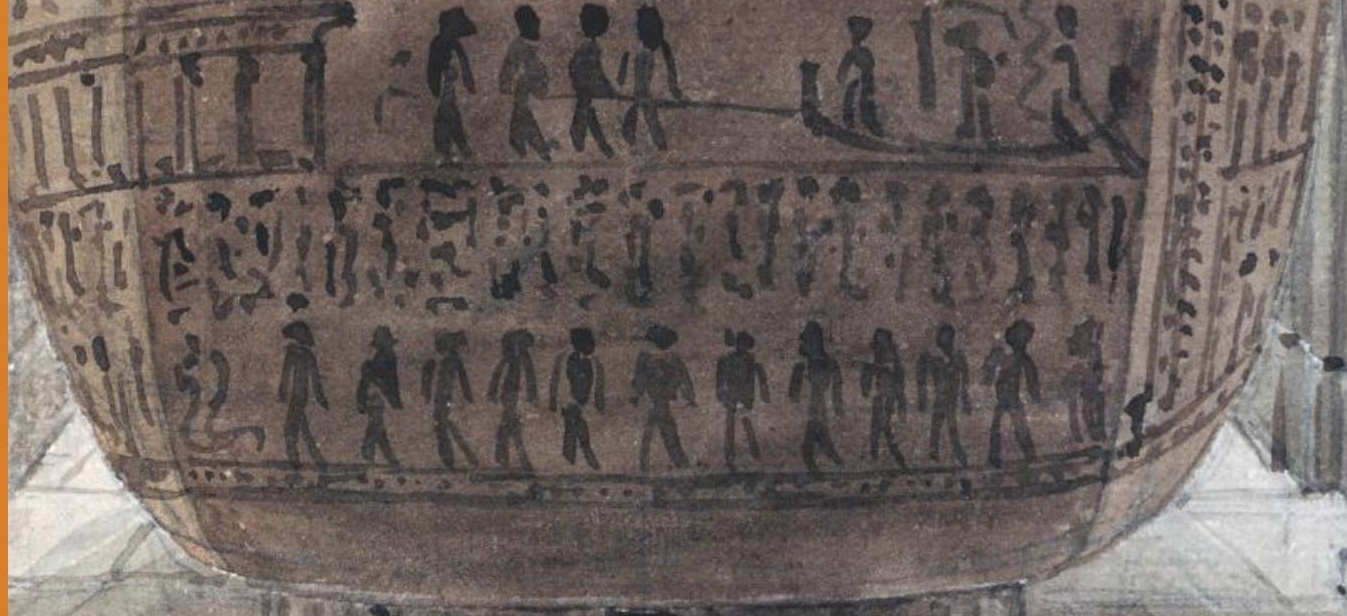

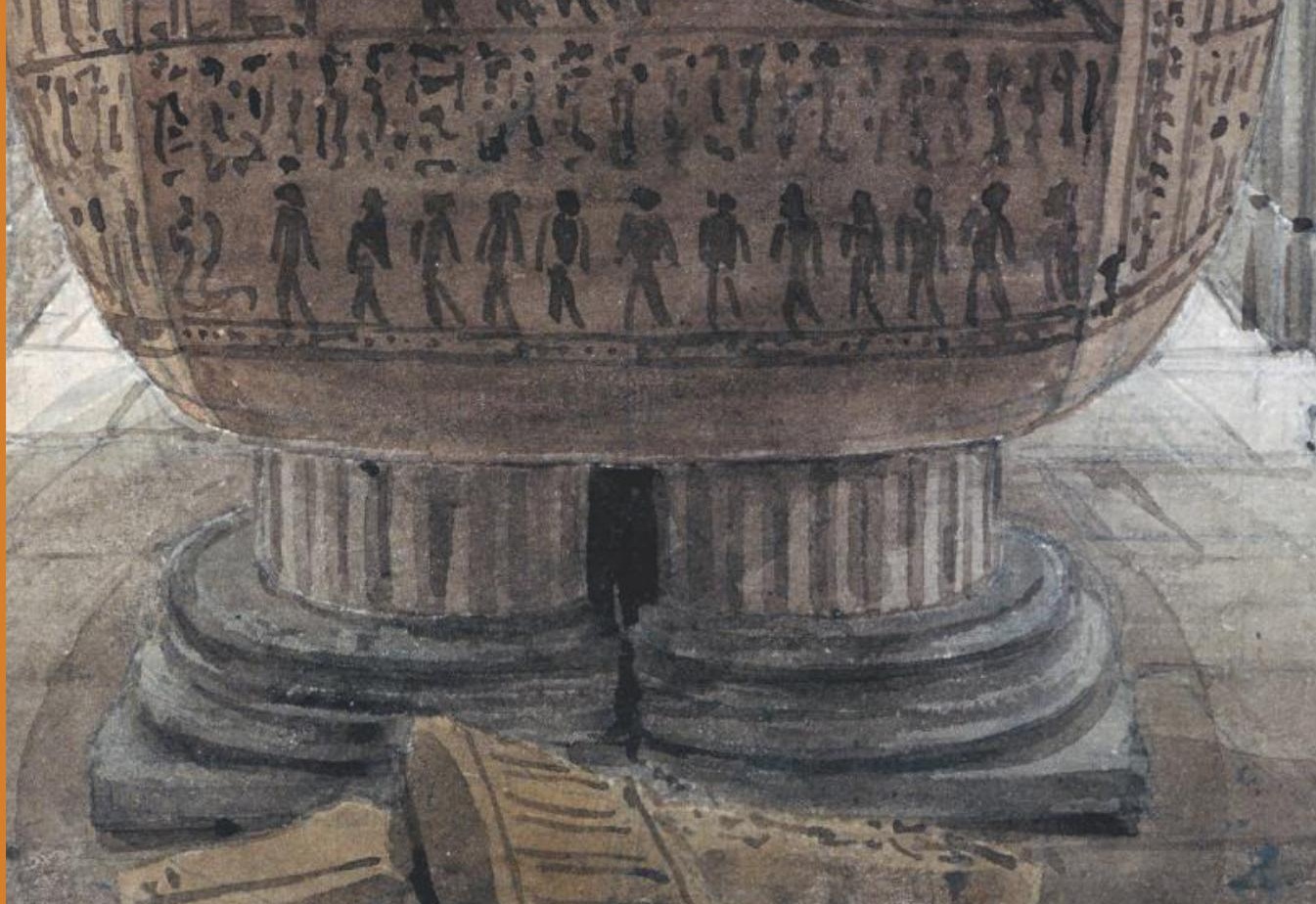

